\title{
The Magellanic Stream: Circumnavigating the Galaxy
}

\author{
Elena D'Onghia, ${ }^{1}$ and Andrew J. Fox, ${ }^{2}$ \\ ${ }^{1}$ Astronomy Department, University of Wisconsin, 475 N Charter Street, \\ Madison, WI, 53706; edonghia@astro.wisc.edu \\ ${ }^{2}$ Space Telescope Science Institute, 3700 San Martin Drive, Baltimore, MD, \\ 21218; afox@stsci.edu
}

This article's doi:
$10.1146 /(($ please add article doi $))$

\section{Keywords}

Magellanic Clouds; galaxy interactions, tidal stripping, circumgalactic gas, gas kinematics, accretion, dynamics

\section{Abstract}

The Magellanic Clouds are surrounded by an extended network of gaseous structures. Chief among these is the Magellanic Stream, an interwoven tail of filaments trailing the Clouds in their orbit around the Milky Way. When considered in tandem with its Leading Arm, the Stream stretches over 200 degrees on the sky. Thought to represent the result of tidal interactions between the Clouds and ram-pressure forces exerted by the Galactic corona, its kinematic properties reflect the dynamical history of the closest pair of dwarf galaxies to the Milky Way. The Stream is a benchmark for hydrodynamical simulations of accreting gas and cloud/corona interactions. If the Stream survives these interactions and arrives safely in the Galactic disk, its cargo of over a billion solar masses of gas has the potential to maintain or elevate the Galactic star formation rate. In this article, we review the current state of knowledge of the Stream, including its chemical composition, physical conditions, origin, and fate. We also review the dynamics of the Magellanic System, including the proper motions and orbital history of the Large and Small Magellanic Clouds, the first-passage and second-passage scenarios, and the evidence for a Magellanic Group of galaxies. 


\section{Contents}

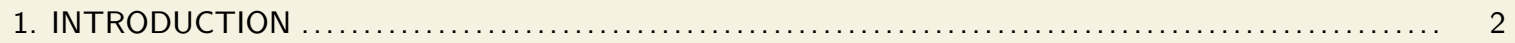

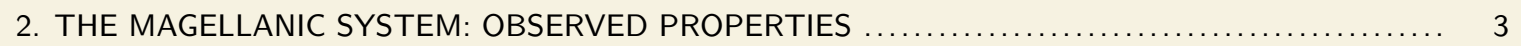

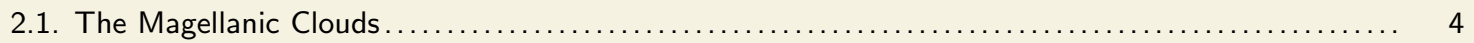

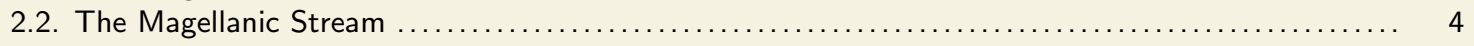

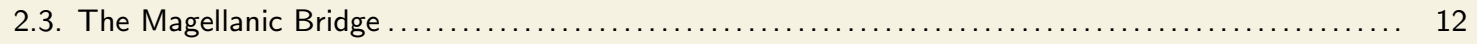

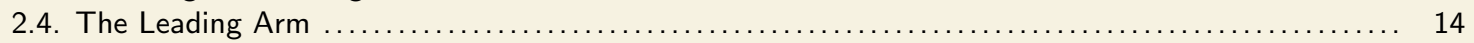

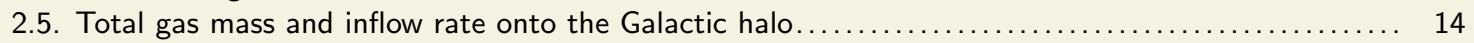

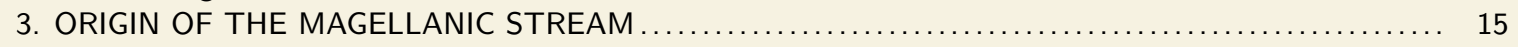

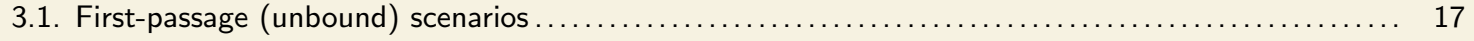

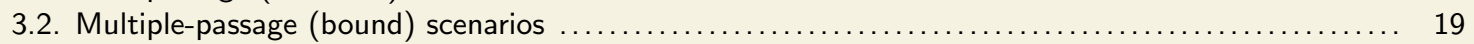

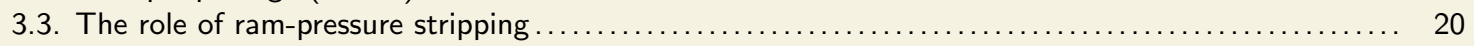

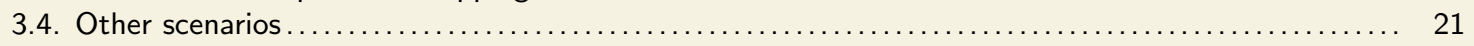

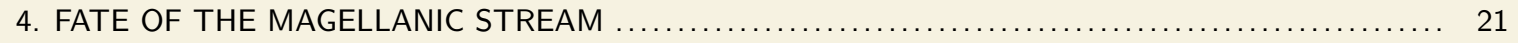

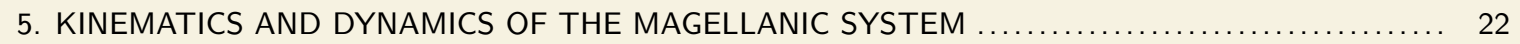

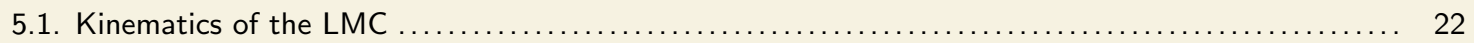

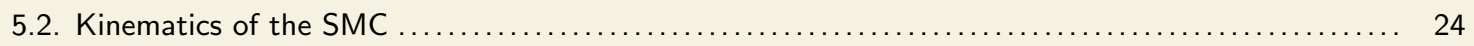

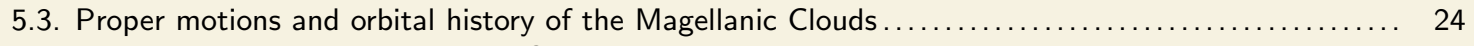

5.4. Proper motions from the Hubble Space Telescope................................... 25

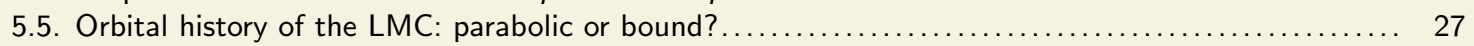

5.6. The Magellanic Group ................................................... 29

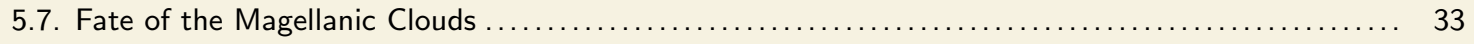

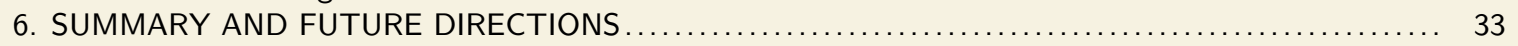

\section{INTRODUCTION}

"If we worked on the assumption that what is accepted as true really is true, then there would be little hope for advance" - Orville Wright, aviation pioneer

The Magellanic Stream, an extended tail of neutral and ionized gas trailing the Magellanic Clouds in their orbit around the Milky Way (MW), was discovered in several radio surveys of the southern sky. The first hints of its existence were provided by Dieter (1965), who mapped high Galactic latitude regions in $21 \mathrm{~cm}$ emission and found a population of high-velocity clouds (HVCs) near the south Galactic pole. Other H I clouds were soon found in similar regions of the sky (Hulsbosch \& Raimond 1966, Hulsbosch 1968, Dieter 1971), and while the distance to these clouds was at that time unknown, the deeper 21 cm studies of van Kuilenburg (1972) and Wannier \& Wrixon (1972) revealed they formed a coherent linear structure. Soon afterwards Mathewson et al. (1974) used the Parkes radio telescope to trace the structure back to the Magellanic Clouds, and coined the term Magellanic Stream (hereafter the Stream). The Mathewson et al. paper is often credited with the discovery of the Stream; in hindsight, all the H I papers cited above had detected parts of it, but Mathewson et al. were the first to demonstrate its Magellanic source. Mathewson et al. 1974 further detected a counterpart to the Stream on the other side of the Magellanic Clouds (the Leading Arm) that extends across the Galactic plane into positive Galactic latitudes, and suggested the extension was a continuation of the same structure. The Magellanic nature of the Leading Arm was later confirmed by kinematic (Putman et al. 1998) and metallicity (Lu et al. 1998) measurements. 
Table 1 The Magellanic System: Mass Budget

\begin{tabular}{|c|c|c|c|c|c|c|}
\hline Property & LMC & $\mathrm{SMC}$ & Stream & Bridge & Leading Arm & Refs. \\
\hline Stellar mass & $3 \cdot 10^{9} \mathrm{M}_{\odot}$ & $3 \cdot 10^{8} \mathrm{M}_{\odot}$ & $\ldots$ & $1.5 \cdot 10^{4} \mathrm{M}_{\odot}{ }^{a}$ & $\ldots$ & $(1,2,3)$ \\
\hline Halo mass & $1.7 \cdot 10^{10} \mathrm{M}_{\odot}^{b}$ & $2.4 \cdot 10^{9} \mathrm{M}_{\odot}{ }^{c}$ & $\ldots$ & $\ldots$ & $\ldots$ & $(4,2)$ \\
\hline H I gas mass & $4.4 \cdot 10^{8} \mathrm{M}_{\odot}$ & $4.0 \cdot 10^{8} \mathrm{M}_{\odot}$ & $2.7 \cdot 10^{8} \mathrm{M}_{\odot}^{d}$ & $1.8 \cdot 10^{8} \mathrm{M}_{\odot}$ & $3.0 \cdot 10^{7} \mathrm{M}_{\odot}$ & (5) \\
\hline H II gas mass & $\ldots$ & $\ldots$ & $\approx 10^{9} \mathrm{M}_{\odot}{ }^{e}$ & $0.7-1.7 \cdot 10^{8} \mathrm{M}_{\odot}^{f}$ & $\sim 10^{8} \mathrm{M}_{\odot}{ }^{e}$ & $(6,7)$ \\
\hline
\end{tabular}

References: (1) van der Marel et al. 2002; (2) Stanimorović et al. 2004; (3) Harris 2007, (4) van der Marel

\& Kallivayalil 2014; (5) Brüns et al. 2005; (6) Fox et al. 2014; (7) Barger et al. 2013.

a Assuming a Kroupa IMF and a $10 \mathrm{Gyr}$ old stellar population.

${ }^{b}$ Out to a radius $8.7 \mathrm{kpc}$; total value depends on tidal truncation of LMC halo.

${ }^{c}$ Dynamical mass based on H I rotation curve. See also Bekki \& Stanimirović 2009.

${ }^{d}$ Mass summed over MS regions I-IV and Interface Region, which we treat as part of Stream. Assumes a distance $d=55 \mathrm{kpc}$

${ }^{e}$ Based on UV absorption. Includes warm-ionized $\left(\sim 10^{4} \mathrm{~K}\right)$ and hot-ionized $\left(\sim 10^{5} \mathrm{~K}\right)$ contributions.

$f$ Based on $\mathrm{H} \alpha$ emission. Includes warm-ionized contribution.

Since this initial phase of discovery and characterization, other methods of studying the Stream have become available, notably ultraviolet (UV) and optical absorption-line spectroscopy of background sources, $\mathrm{H} \alpha$ emission-line spectroscopy, $N$-body modeling, and hydrodynamical simulations. The UV and optical spectroscopic studies are complementary to the radio studies since they address the ionized gas in the Stream. At the same time, successively more sensitive radio surveys at $21 \mathrm{~cm}$ have been completed, bringing the filamentary and fragmented structure of the Stream into focus.

Together these approaches have revolutionized our understanding of the Stream. We now know it is a bifurcated, multi-phase, fragmented, massive gaseous structure that originated in both Magellanic Clouds. It has become a benchmark for the study of gas-transport processes in the halo of an $L_{*}$-galaxy, and is the only known gaseous tidal stream in the vicinity of the MW, where stellar tidal streams are more common. It dominates all the other gaseous high-velocity clouds (HVCs) in the Galactic halo, both in terms of gas mass and inflow rate, and therefore understanding the Stream is essential to a global picture of the Galaxy's circumgalactic medium. Its age and location on the sky constrain dynamical models of the Magellanic Clouds and the total mass of the MW. These many independent reasons have turned the attention of various groups of astronomers onto the Stream. In this review article we synthesize current knowledge and lay out a path for future studies. We also review the subject of Magellanic Dynamics, which is closely related since the Stream's location traces out the past orbits of the LMC and SMC.

We use the term The Magellanic System to include the LMC, SMC, and the three notable gaseous structures surrounding them: the Stream, Bridge, and Leading Arm. The masses of the various components of the Magellanic System are given in Table 1. Note that all gas masses are given assuming a distance $d=55 \mathrm{kpc}$, but will scale as $d^{2}$.

LA: Leading Arm

MB: Magellanic Bridge

MS: Magellanic

Stream

MW: Milky Way

\section{THE MAGELLANIC SYSTEM: OBSERVED PROPERTIES}

In this section the basic observational properties of the Magellanic System are described, starting with an overview of the Magellanic Clouds (Sect. 2.1). We then cover the Magellanic Stream (Sect. 2.2), including its structure and morphology, chemical abundance patterns, dust properties, and ionized gas content. We then present an overview of the Magellanic Bridge (Sect. 2.3) and Leading Arm (Sect. 2.4. Finally we discuss the total mass and mass-flow rate of Magellanic gas in Sect. 2.5 


\subsection{The Magellanic Clouds}

The Large and Small Magellanic Clouds (LMC/SMC) are dwarf irregular galaxies in the southern hemisphere. Both are satellite galaxies of the MW, visible to the naked eye in the constellations Dorado (LMC) and Tucana (SMC). They have therefore been known since ancient times, but adopt their name from Ferdinand Magellan who observed them on his expedition in 1519-1522. The Clouds are the closest pair of galaxies to the MW and a unique location for studying galaxy-formation processes. The Clouds and the MW form the nearest ensemble of interacting galaxies, representing the best opportunity anywhere for studying satellite-satellite and satellite-host galaxy gravitational interactions.

The LMC, at a distance of $50 \pm 1$ kpc (Pietrzyński et al. 2013, Walker 2012), is the prototypical Barred Magellanic Spiral, a population of galaxies with peculiar morphological properties. These include an asymmetric stellar bar, which may be off-centered from the dynamical center of the galaxy, a single looping spiral arm, and often a large star-forming complex at one end of the bar (de Vaucouleurs \& Freeman 1972). The LMC's stars are concentrated into a flat disk tilted at an inclination $i=45^{\circ}$ with respect to the plane of the sky, and it has a luminosity one-tenth that of the MW (Sparke \& Gallagher 2000). Its currentday metallicity, as determined from massive main-sequence stars, is $[\mathrm{Z} / \mathrm{H}]=-0.31 \pm 0.04$, i.e. $\approx 0.5$ solar (Rolleston et al. 2002; see also Russell \& Dopita 1992). The total LMC mass (out to $8.7 \mathrm{kpc}$ ) is $1.7 \cdot 10^{10} \mathrm{M}_{\odot}$ (van der Marel \& Kallivayalil 2014; see Table 1), although it is unclear how extended the halo of LMC is, so the total mass could be considerably higher.

The SMC, at $61 \pm 1 \mathrm{kpc}$ (Hilditch et al. 2005, Graczyk et al. 2014), is the closest dwarf irregular galaxy, and an excellent benchmark for studying the evolution of late-type dwarf galaxies. It has an elongated (cigar-shaped) structure seen end-on. The SMC's current-day metallicity is $\approx 20 \%$ solar derived from H II regions and young stars (Russell \& Dopita 1992 ), although the red giant population has $[\mathrm{Fe} / \mathrm{H}]=-0.99 \pm 0.01$, i.e. $10 \%$ solar (Dobbie et al. 2014). The total SMC mass is estimated from rotation curves to be $2.4 \cdot 10^{9} \mathrm{M}_{\odot}$ (Stanimirović et al. 2004), at the upper limit of the mass range of this class of galaxies (Tolstoy et al. 2009). Due to its strong gravitational interaction with the LMC, the SMC exhibits a complex morphology and dynamics, with the gas behaving differently from the stellar populations. The large-scale H I distribution shows an irregular and asymmetric morphology, with prominent features including the visual appearance of a bar, possible extensions to the bar similar to arms, and a "wing" connected by a bridge of gas (Caldwell \& Coulson 1986; Stanimirović et al. 2004). All of these features might be signs of recent tidal interactions with the LMC (Maragoudaki et al. 2001). The small-scale H I distribution is characterized by numerous arcs, filaments, and expanding shells (Stanimirović et al. 1999). The old and young stellar populations in the SMC have different spatial distributions (e.g. Gardiner \& Hatzidimitriou 1992). Young stars with ages $\leq 200 \mathrm{Myr}$ have a distribution that follows the H I gas (Zaritsky et al. 2000), whereas the old stars with ages $\geq 1$ Gyr show a regular spheroidal distribution (Cioni et al. 2000, Harris \& Zaritsky 2006).

The fact that the Magellanic Clouds are so well studied and characterized provides clear advantages for understanding the Stream, since we can compare the kinematics, chemistry, and dust content of the Stream with the corresponding properties of its parent galaxies, and thereby refine our understanding of the Stream's origin.

\subsection{The Magellanic Stream}

$4 \quad$ D'Onghia and Fox 


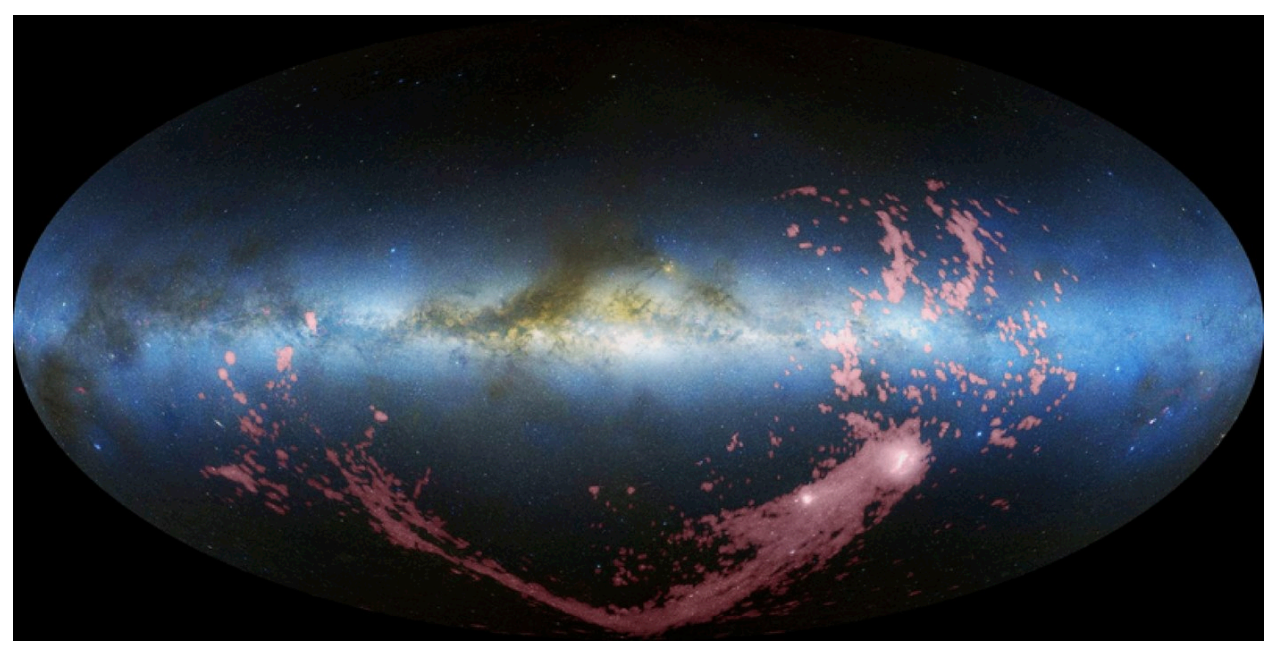

Figure 1

The Magellanic Stream (pink) displayed in Galactic coordinates using an all-sky Hammer-Aitoff projection centered on the Galactic Center (image credit Nidever et al. 2010, NRAO/AUI/NSF, Mellinger 2009, Leiden-Argentine-Bonn Survey, Parkes Observatory, Westerbork Observatory, Arecibo Observatory). The Magellanic Bridge between the LMC and SMC and the Leading Arm are clearly visible, as is the extended fragmentation across the Stream.

2.2.1. The neutral gas: structure and morphology. The Stream was discovered and characterized in H I $21 \mathrm{~cm}$ emission, and so we have a detailed understanding of the spatial structure and morphology of the neutral gas. Our knowledge has progressed hand-in-hand with successive generations of radio telescopes. Major $21 \mathrm{~cm}$ surveys covering parts or all of the Stream include those by Mathewson et al. (1974, 1977), Bajaja et al. (1985), Hulsbosch \& Wakker (1988), Morras et al. (2000), Lockman et al. (2002), Putman et al (2003b), Brüns et al. (2005), Kalberla et al. (2005), Stanimirović et al. (2008), Nidever et al. (2008, 2010), and McClure-Griffiths et al. (2009) The full size of the Stream can be seen in Figure 1 (Nidever et al. 2010), which shows its extent on an all-sky map.

Mathewson et al. (1977) identified six horseshoe-shaped concentrations of gas along the Stream, which they named MS I-VI with increasing angular separation from the Clouds. The gas surface density decreases monotonically from MS I and to MS VI. This naming convention is still seen in the literature, but the higher sensitivity of the more recent surveys has revealed diffuse gas between the dense clumps. We now know that the Stream consists of an almost linear central body surrounded by a significant number of small-scale fragmentary structures. The small-scale structure is evident across the Stream, from its tip farthest from the Magellanic Clouds to its source where it connects with them (Mirabel et al. 1979, Haynes 1979, Mirabel 1981, Wayte 1989, Wakker et al. 2002, Stanimirović et al. 2002, 2008, Putman et al. 2003b, Westmeier \& Koribalski 2008). The Leading Arm (LA) also shows substantial small-scale structure (Putman et al. 1998). The extent of the fragmentation is revealed by the work of For et al. (2014), who cataloged 251 discrete clouds and filaments across the Stream. In Figure 2 we show an overview of the H I emission from the Stream, with the various components labeled.

Furthermore, the central body of the Stream is bifurcated into two main strands or 


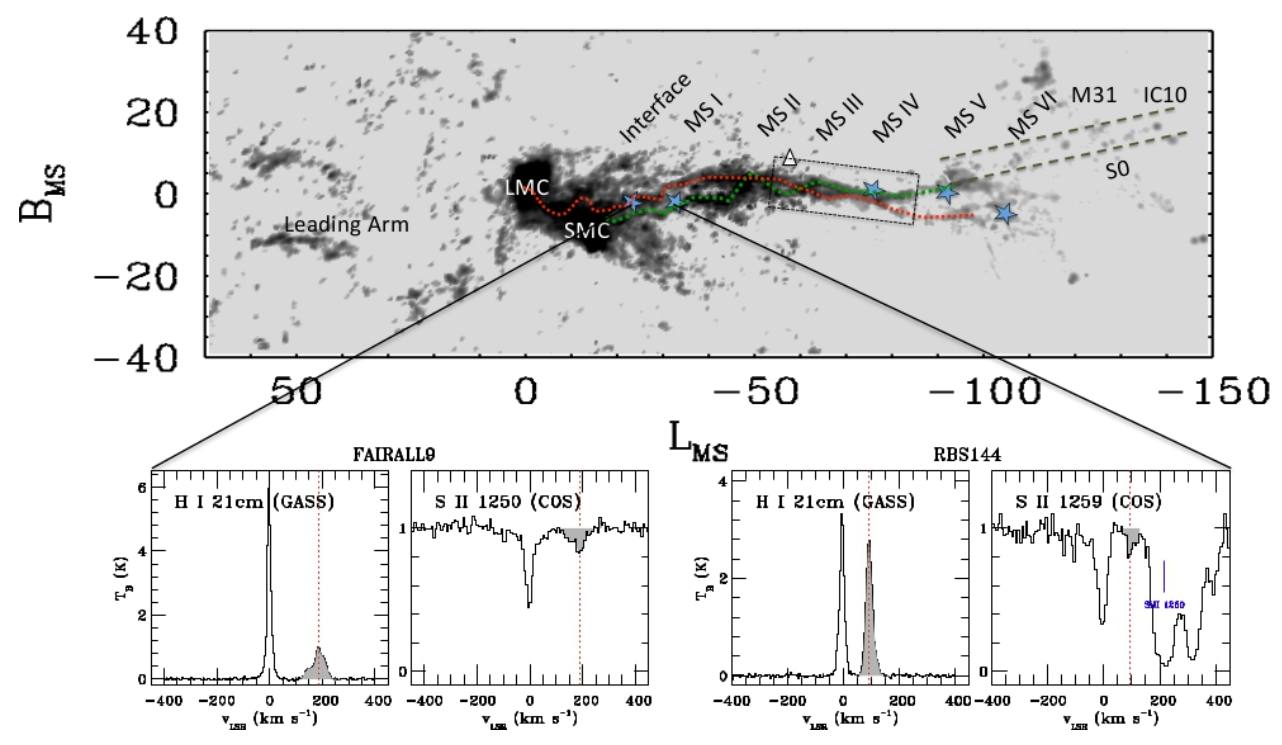

Figure 2

Top: Overview of the Magellanic System (Hammer et al. 2015), showing H I data from the GASS survey (McClure-Griffiths et al. 2009) plotted in the Magellanic coordinate system of Nidever et al. (2010). The main components of the system are labeled. The red and green dotted lines denote the LMC and SMC filaments, respectively, and the blue stars show directions where UV metal-abundance measurements are available (Fox et al. 2013). Bottom: $21 \mathrm{~cm}$ and UV spectra of the Stream in two directions, one passing through the SMC filament (RBS 144) and one passing through the LMC filament (Fairall 9). The relative strength of the H I emission and S II absorption provides a direct measurement of the metallicity. The two filaments have sulfur abundances differing by a factor of five, confirming their different origin (Fox et al. 2013; Richter et al. 2013).

filaments (see Figure 2), as was first recognized by Cohen (1982) and Morras (1983). Deeper observations of the Stream's bifurcated structure were presented by Putman (2000) and Putman et al. (2003b), using data from the H I Parkes All Sky Survey (HIPASS; Barnes et al. 2001). The bifurcation of the Stream has also been detected kinematically (Nidever et al. 2008), in that the two strands have velocities that trace back to different origins (with one connecting to the LMC), and chemically (Fox et al. 2013, Richter et al. 2013), in that the two strands show different chemical abundance patterns, one resembling the SMC pattern and the other resembling the LMC pattern. The Stream is therefore an interwoven tail of two filaments.

The kinematic bifurcation found by Nidever et al. (2008) was derived from a Gaussian decomposition of the Leiden-Argentine-Bonn (LAB) $21 \mathrm{~cm}$ emission data on the Stream (Kalberla et al. 2005). Using position-velocity diagrams, Nidever et al. showed that one filament of the Stream can be traced back to the LMC, not the SMC, as had traditionally been assumed. Furthermore, this LMC filament connects to a region of the LMC known as the southeast H I over-density (SEHO), which includes the starburst region 30 Doradus. This provides an important and hitherto-unknown connection between the Stream and starformation activity within the Clouds, as if the LMC strand is a smoke trail from a Magellanic chimney. Nidever et al. report that the other filament is not conclusively connected to either 
Magellanic Cloud, but its chemical properties have subsequently been shown to indicate an SMC origin (see Sect. 2.2.2.

Many bulk properties of the neutral gas in the Stream are well known. The Stream has a total H I mass of $2.7 \cdot 10^{8} \mathrm{M}_{\odot}$ (for a distance $d=55 \mathrm{kpc}$, and including the "Interface Region" near the SMC; Brüns et al. 2005). When considered in tandem with the Bridge and Leading Arm, the total H I mass in the Magellanic System is $5.0 \cdot 10^{8} \mathrm{M}_{\odot}$ and covers a total angular extent of 2700 square degrees on the sky down to a limiting column density $N(\mathrm{H}$ I) $=10^{18.0} \mathrm{~cm}^{-2}$ (Nidever et al. 2010). There is a fairly smooth column density gradient along the Stream (Mathewson et al. 1974, Putman et al. 2003b, Nidever et al. 2010) which can be written as $N(\mathrm{H} \mathrm{I})=5.9 \cdot 10^{21} \exp \left(l_{\mathrm{MS}} / 19.3^{\circ}\right) \mathrm{cm}^{-2}$, where $l_{\mathrm{MS}}$ is the Magellanic Longitude, the angular distance from the LMC along the main axis of the Stream (Nidever et al 2010; see also Wakker 2001). The velocity of the Stream in the Local Standard of Rest (LSR) varies from $+180 \mathrm{~km} / \mathrm{s}$ near the Clouds to $-450 \mathrm{~km} / \mathrm{s}$ near its tip.

Nidever et al. (2008) reported periodic, sinusoidal undulations in the H I Stream; they suggest these could be the imprint of the LMC rotation curve, related to the position of the SEHO in the LMC at the time the gas was stripped. In this scenario the drift rate of the Stream away from the LMC is $\sim 49 \mathrm{~km} / \mathrm{s}$ and its age is $\sim 1.7 \mathrm{Gyr}$, in good agreement with tidal age estimates of the Stream (Besla et al. 2010, 2012). With the more spatiallyextended Stream reported by the deeper H I measurements of Nidever et al. (2010), the age increases to $\sim 2.5$ Gyr. Putman et al. (2003b) had already noticed the double-helix morphology of the Stream and suggested it may be related to the binary motion of the LMC and SMC.

The typical H I line widths in the Stream are 18-25 km/s (FWHM; Mathewson \& Ford 1984; Hsu et al. 2011), which under the assumption of thermal broadening correspond to gas temperatures of 7000-14000 K, although there are regions with line widths as narrow as $3 \mathrm{~km} / \mathrm{s}$ (Kalberla \& Haud 2006, Stanimirović et al. 2008). Regions of cooler gas in the Stream were detected by Matthews et al. (2009), via $21 \mathrm{~cm}$ absorption studies. They detected a cool cloud with an inferred spin temperature of $70 \mathrm{~K}$ toward one continuum source, but failed to detect cool gas toward three other sources, indicating the cool phase has a low covering fraction. Furthermore, a UV detection of $\mathrm{H}_{2}$ absorption by Richter et al. (2001) toward the QSO Fairall 9 with a total $\mathrm{H}_{2}$ column $\log N\left(\mathrm{H}_{2}\right)=16.40_{-0.53}^{+0.28}$ and a molecular fraction $f\left(\mathrm{H}_{2}\right)=5 \cdot 4 \cdot 10^{-4}$ provides evidence for a molecular phase. No other sightline through the Stream to a UV-bright continuum source has yielded an $\mathrm{H}_{2}$ detection, indicating a low covering fraction for the molecular phase, although there are $\mathrm{H}_{2}$ detections in the Leading Arm (Sembach et al. 2001) and Bridge (Lehner 2002), and recently Murray et al. (2015) reported $\mathrm{HCO}^{+}$absorption from the leading edge of the Bridge. The cool $\mathrm{H}$ I, $\mathrm{H}_{2}$, and $\mathrm{HCO}^{+}$detections demonstrate the multi-phase nature of the gas in the Stream. This is also revealed by the many forms of ionized gas that are present, as we discuss in Section 2.2 .4

The length of the known Stream has grown with time as more diffuse gas clouds have been discovered. Nidever et al. (2010) confirmed that the numerous small clouds in the "northern extension region" near the Galactic plane (defined by Braun \& Thilker 2004) indeed belong to the Stream. This means that the Stream and Leading Arm together cover over $200^{\circ}$ in length on the sky.

2.2.2. Chemical abundance patterns. The presence of metals in the Stream was first demonstrated by Songaila (1981), who used optical spectroscopy of the background quasar Fairall 9 
to detect $\mathrm{Ca}$ II and $\mathrm{Na}$ I absorption at the Stream's velocity. These lines trace cool clumps of dense neutral gas, and have since been reported in other Stream directions (West et al. 1985, Fox et al 2013, Richter et al. 2013). As Ca II and Na I fall in the optical, they can be observed at higher spectral resolution and higher sensitivity than is possible with UV lines that require space-based observations. However, both Ca II and Na I are non-dominant (trace) ionization stages, and so these lines are of limited use for metallicity measurements. Nonetheless, observing them helps to reveal the clumpiness and velocity structure of the cool gas.

Measuring the metallicity of the Stream requires a combination of metal-line and $\mathrm{H} \mathrm{I}$ observations. UV absorption-line spectra provide the metal-line column densities and 21 $\mathrm{cm}$ emission-line spectra provide the H I column densities. Until the installation of the Cosmic Origins Spectrograph (COS) on the Hubble Space Telescope in 2009, only a very limited number of AGN lying behind the Stream were bright enough for UV spectroscopic observations (Savage et al. 2000). One of the few exceptions is the QSO Fairall 9, which lies behind the head of the Stream close to the LMC. Lu et al. (1994) measured two constraints on the Stream's metallicity along this sightline: a silicon abundance $(\mathrm{Si} / \mathrm{H}) \geq 0.2$ solar and a sulfur abundance $(\mathrm{S} / \mathrm{H}) \leq 0.3$ solar. A metallicity measurement (rather than a limit) in the Fairall 9 sightline was made by Gibson et al. (2000), who found $(\mathrm{S} / \mathrm{H})=0.28_{-0.10}^{+0.17}$ solar, consistent with a Magellanic origin, but this measurement does not distinguish between LMC and SMC scenarios. In the tip of the Stream (farthest from the Magellanic Clouds), Fox et al $(2010)$ measured $(\mathrm{O} / \mathrm{H})=0.10 \pm 0.02$ solar, the first indication of a metallicity below the current day SMC abundance of $\approx 0.2$ solar.

Recent work on the chemical abundances of the Stream using HST/COS has found that the two principal filaments show different chemical abundances. The filament shown by Nidever et al (2008) to connect kinematically to the LMC indeed shows LMC-like abundances ( $\approx 0.5$ solar; Gibson et al. 2000; Richter et al. 2013). The other filament shows SMC-like abundances in multiple directions $(\approx 0.1$ solar toward RBS 144 , NGC 7714, and NGC 7469; Fox et al. 2013). These COS measurements were made using the relatively undepleted elements sulfur or oxygen, via the $(\mathrm{S} \mathrm{II} / \mathrm{H} \mathrm{I})$ or $(\mathrm{O} \mathrm{I} / \mathrm{H} \mathrm{I})$ ratios, so are not thought to be significantly affected by dust depletion. They were also made in directions of high $\mathrm{H}$ I column $N(\mathrm{H} \mathrm{I})>10^{20} \mathrm{~cm}^{-2}$, which are predominantly neutral and self-shielding, so ionization corrections are expected to be small. The significance of these measurements is the confirmation that both Magellanic Clouds contributed gas to the Stream.

The finding that the measured abundance of the "SMC filament" is 0.1 solar, lower than the mean current-day SMC abundance of $\approx 0.2$ solar (Russell \& Dopita 1992) could have several explanations. First, it could indicate that the gas was stripped in the past, when the mean SMC metallicity was lower. Indeed the SMC's age-metallicity relation (Pagel \& Tautvaisiene 1998; Harris \& Zaritsky 2004; Cignoni et al. 2013) indicates that the mean SMC metallicity was $\approx 0.1$ solar $\approx 2-2.5 \mathrm{Gyr}$ ago, in agreement with the time when tidal models find that the main body of the Stream was formed (Besla et al. 2010, see Section 5]. The timescales for this model are therefore self-consistent: the gas has the right metallicity for its tidal age. Alternatively, the low measured abundance could indicate that the Stream was stripped from outer, less metal-enriched regions of the SMC, since peripheral gas would be more loosely bound gravitationally and hence easier to strip. However, the SMC does not show a strong radial chemical abundance gradient (Pagel et al. 1978, Cioni 2009), at least at the present time. A final possibility is that some degree of metal mixing between the cool gas and the surrounding hot gas has diluted the Stream, 
lowering its initial metallicity, although this is hard to quantify, since metal mixing between different gas phases is a poorly understood process, and the metallicity of the hot gas phase is itself poorly known.

Further information on the chemical enrichment of the Stream is provided by observations of the compact high-velocity cloud (CHVC) 224.0-83.4-197 located close $\left(<0.7^{\circ}\right.$ separation) to the Stream along the line of sight to the bright AGN Ton S210. The CHVC has a metallicity consistent with 0.1 solar (Sembach et al. 2002, Richter et al. 2009), with the latest measurement being $[\mathrm{O} / \mathrm{H}]=-1.12 \pm 0.22$ (Kumari et al. 2015). Based on the match between this cloud's metallicity and that of the nearby filaments of the main Stream, Kumari et al. suggest the cloud may be an outlying fragment of the Stream. This is the only small outlying cloud whose metallicity has been shown to match the Stream itself; it is interesting that no obvious dilution of the gas (which would have lowered the metallicity) has occurred.

2.2.3. Dust content. The relative abundance of refractory elements, which deplete onto dust grains, and volatile ones, which do not, provides an empirical measurement of the dust depletion level in the Stream. The earliest application of this technique was by $\mathrm{Lu}$ et al. (1994), who found little evidence for dust in the Fairall 9 direction (through the LMC filament) based on a measurement $(\mathrm{Si} / \mathrm{S})>0.6$ solar. However, in the Leading Arm toward the Seyfert galaxy NGC 3783, Lu et al (1998) found a highly super-solar S/Fe ratio ( $7.6 \pm 2.2$ solar), indicative of dust because iron is depleted onto dust grains but sulfur is not. Sembach et al (2001) came to a similar conclusion based on a Far-Ultraviolet Spectroscopic Explorer (FUSE) spectrum of the same AGN, and further concluded that the dust grains in the Leading Arm had been processed, with the grain mantles modified or stripped to expose the grain cores.

In the SMC filament where the overall metallicity is 0.1 solar, Fox et al. (2013) found evidence for dust in the form of sub-solar $\mathrm{Si} / \mathrm{S}, \mathrm{Al} / \mathrm{S}$, and $\mathrm{Fe} / \mathrm{S}$ ratios, with $\delta(\mathrm{Si}) \approx-0.6$, $\delta(\mathrm{Al}) \approx-0.7$, and $\delta(\mathrm{Fe}) \approx-0.6$, where $\delta(\mathrm{X})=[\mathrm{X} / \mathrm{S}]-[\mathrm{X} / \mathrm{S}]_{\odot}$, i.e. the depletion relative to sulfur. This demonstrates that the dust grains survived the process(es) that formed the Stream and also survive in the incident ionizing radiation field. In the LMC filament toward Fairall 9, where $(\mathrm{S} / \mathrm{H})=0.5$ solar, Richter et al. (2013) found a low $[\alpha / \mathrm{N}]$ ratio of -0.85 dex, indicating the gas there is chemically young, i.e. the $\alpha$-elements have been released by Type II supernovae but $\mathrm{N}$ has not yet been released, since that requires a longer $(\approx 250 \mathrm{Myr})$ timescale until intermediate-mass stars evolve into the AGB phase. Richter et al. find $\delta(\mathrm{Al}) \approx-0.62, \delta(\mathrm{Si}) \approx-0.27$, and $\delta(\mathrm{Fe}) \approx-0.56$, similar to the depletions in the SMC filament, despite the factor of five difference in the overall metallicity in the two directions.

Combining the depletions with the H I column densities yields the gas-to-dust ratios (G/D) in the Stream. These were calculated for two sightlines by Fox et al. (2013). In the SMC filament toward RBS144, the G/D ratio normalized to the local Galactic ISM value is $(\mathrm{G} / \mathrm{D})_{\text {norm }}=19_{-6}^{+10}$. In the LMC filament toward Fairall $9,(\mathrm{G} / \mathrm{D})_{\text {norm }}=3.3_{-0.5}^{+0.5}$, a factor of $\approx 6$ lower than in the SMC filament. Since the LMC filament has a metallicity a factor of $\approx 5$ higher than the SMC filament, the dust mass per unit gas mass scales almost linearly with the metallicity.

An independent approach for studying the dust content of the Stream is to look for thermal far-IR emission in regions of high H I column density. Fong et al. (1987) conducted such a search for regions of the Stream near the south Galactic pole, and found no correlation with the IRAS 100-micron flux, indicating that the Stream has a lower dust-to-gas ratio 
than interstellar gas in the MW, consistent with the UV-depletion measurements.

2.2.4. Ionized gas. Two principal ionization processes operate on the gaseous Stream: photoionization and collisional ionization (we ignore the potential contribution from cosmic rays). UV absorption-line observations (Lu et al. 1994, 1998; Sembach et al. 2001, 2003; Fox et al. 2005, 2010, 2013, 2014; Richter et al. 2013, Kumari et al. 2015) reveal that both low-ionization and high-ionization phases of gas are present in the Stream, with distinct kinematic properties. The high ions C IV, Si IV, and O VI often show broader line widths and offset velocity centroids (by $<20 \mathrm{~km} / \mathrm{s}$ ) compared to the low ions such as C II, Si II, Fe II, and Al II, but still show absorption in the same general regions of velocity space (such high-ion/low-ion profile differences are a common feature of circumgalactic environments). This indicates that the ionized gas in the Stream is multi-phase, i.e. it contains regions of different gas density and temperature.

The low-ion phase can be successfully modeled as a photoionized plasma illuminated by Lyman-continuum photons. These photons have multiple sources, including the MW disk, the Magellanic Clouds, and the extragalactic UV background. The MW radiation itself has contributions from several components, including O-B stars, planetary nebulae, and potentially the central supermassive black hole. The relative contribution from those sources has been modeled by Bregman \& Harrington (1986), Bland-Hawthorn et al. (1999, 2013) and Fox et al. (2005, 2014). Calculating these radiation fields allows photoionization models to be generated for the metal-line column densities measured in the Stream, which in turn constrains the physical conditions in the gas, such as gas density, pressure, and ionized-hydrogen column density. The $\mathrm{H}^{+}$column can be combined with constraints on the cross-sectional area of the Stream to yield the ionized gas mass. The photoionization models are therefore crucial for understanding the overall properties of the low-ion phase. Representative values from photoionization models for the gas density, thermal pressure, and line-of-sight cloud size are $\log \left(n / \mathrm{cm}^{-3}\right)=-1.8$ to $-2.2, P / k=30-250 \mathrm{~cm}^{-3} K, l_{l o s}=0.7-$ $20 \mathrm{kpc}$ (Fox et al. 2014).

The high-ion phase is traced by absorption in O VI (Sembach et al. 2003) and C IV and Si IV (Lu et al. 1994, Fox et al. 2010, Richter et al. 2013, Kumari et al. 2015) and is thought to be collisionally ionized. The main argument that collisional ionization is favored over photoionization is that the photoionization models that explain the low-ion column densities under-produce the observed high-ion columns by orders of magnitude. The underproduction is most severe for O VI, where the average Stream column is $\langle\log$ $N(\mathrm{O}$ VI $)\rangle=14.10$ (Sembach et al. 2003). Another way to state the problem is that there are not enough highly energetic $(E>113.6 \mathrm{eV})$ photons to ionize the observed amounts of O VI within reasonable pathlengths (they require cloud sizes of $\sim 100 \mathrm{~s}$ of $\mathrm{kpc}$, which are incompatible with having to fit into a small volume of the Galactic halo). However, photoionization may be an important contributor to the C IV and Si IV, which can be created by photons of $47.9 \mathrm{eV}$ and $33.5 \mathrm{eV}$, respectively, and such photons are expected from the spectra of hot stars as well as the (harder) extragalactic ionizing background.

Since there is clearly (a) cool gas in the Stream and (b) an external medium with which the Stream interacts, it is often argued that the high ions exist in the collisionally-ionized boundary layers between the H I gas and the hot coronal plasma. However, it is unclear what the energy transport mechanism in those layers is; they could be conductively heated, turbulently mixed, and/or shocked. Each of these scenarios has been explored in simulations that track the gas ionization under certain physical conditions (e.g. Gnat \& Sternberg 2009, 
Gnat et al. 2010) or in full hydrodynamic simulations (e.g. Kwak \& Shelton 2010, Kwak et al. 2011). Each model can be tested by comparing the high-ion column density ratios it predicts with those observed in the Stream via UV observations, such as $N(\mathrm{C} \mathrm{IV}) / N(\mathrm{O}$ $\mathrm{VI})$ and $N(\mathrm{Si} \mathrm{IV}) / N(\mathrm{C} \mathrm{IV})$. For one outer-Stream sight line (toward the QSO HE 02264110), Fox et al. (2005) concluded that either turbulent mixing or conductive heating is a viable explanation for the high ions in the Stream. This explanation is also favored for other HVCs in the Galactic halo (Collins et al. 2005; Ganguly et al. 2005), with turbulent mixing layers favored over conductive interfaces in the large dataset presented by Wakker et al. 2012. Nigra et al. (2012) also favor turbulent mixing over conductive heating based on the size of an extended diffuse layer seen around a small clump of the Stream in deep $21 \mathrm{~cm}$ observations.

$\mathrm{H} \alpha$ emission traces the warm ionized gas in the Stream in a complementary manner to the low-ion UV absorption lines. $\mathrm{H} \alpha$ is a recombination line whose intensity scales as density squared, whereas the strength of UV absorption scales linearly with the density. Thus the $\mathrm{H} \alpha$ emission preferentially traces the densest regions of plasma in the Stream. The first measurements of $\mathrm{H} \alpha$ emission from the Stream (Weiner \& Williams 1996) found intensities in the range $0.20-0.37$ Rayleigh ${ }^{1}$. These authors interpreted this emission as the signature of an extended gaseous halo that is ram pressure-stripping the cool gas in the Stream. Putman et al. (2003a) found that the $\mathrm{H} \alpha$ emission is more variable, with intensities between 0.10 and 0.41 Rayleighs. Although the $\mathrm{H} \alpha$ intensity of other (less distant) HVCs has been used as an approximate distance indicator (Bland-Hawthorn et al. 1999, 2002; the $\mathrm{H} \alpha$ intensity scales linearly with the incident Lyman continuum flux, which declines with distance from the Galaxy), the variability of the $\mathrm{H} \alpha$ from the Stream challenges its use as a distance indicator. $\mathrm{H} \alpha$ emission was detected from several small clumps (in the MS IV region) by Yagi et al. (2012), who favored a shock-cascade origin for the H $\alpha$ (BlandHawthorn et al. 2007, Tepper-Garcia et al. 2015) since these clumps lie at the leading edge of a downstream cloud. A more recent survey of 17 Stream directions with the Wisconsin H $\alpha$ Mapper (WHAM; Barger et al. 2015, in prep.) finds that the $\mathrm{H} \alpha$ emission often extends several degrees off the edges of the $\mathrm{H}$ I contours, as if the $\mathrm{H} \alpha$ traces the extended surfaces of the Stream's neutral clouds and filaments.

An alternative possibility is that the $\mathrm{H} \alpha$ emission from the Stream was photo-excited by a burst of Galactic Center (GC) activity (such as a Seyfert flare) $\sim 2$ Gyr ago (BlandHawthorn et al. 2013). In this scenario the Stream was ionized by GC Lyman-continuum photons and is now recombining and emitting $\mathrm{H} \alpha$. The $\sim 2$ Gyr timescale is plausible since (a) it is similar to the estimated age of the Fermi Bubbles that surround the GC and are powered by some form of GC activity ( $\mathrm{Su}$ et al. 2010), and (b) the nuclear wind has been dated to $\sim 2-4$ Gyr based on velocity measurements from UV absorption lines (Fox et al. 2015). The variability of the observed $\mathrm{H} \alpha$ from the Stream makes it difficult to search for enhanced emission in a cone below the south Galactic pole, which is a signature of this GC flare model, but such enhanced ionization might be detected by a sufficient number of UV absorption-line observations of suitably-placed QSOs.

In addition to photoionization from external radiation fields, in situ photons emitted by the cooling hot plasma seen in O VI absorption can also photoionize $\mathrm{H} \alpha$ (see appendix in Wakker et al. 2012). Shock ionization can also contribute to the $\mathrm{H} \alpha$ emission from the Stream (Bland-Hawthorn et al. 2007; Tepper-Garcia et al. 2015). In conclusion, multi-

\footnotetext{
${ }^{1} 1$ Rayleigh is $10^{6} / 4 \pi$ photons $\mathrm{cm}^{-2} \mathrm{sr}^{-1} \mathrm{~s}^{-1}$, or equivalently $1.7 \cdot 10^{-6} \mathrm{erg} \mathrm{cm}^{-2} \mathrm{sr}^{-1} \mathrm{~s}^{-1}$ at $\mathrm{H} \alpha$.
} 
ple ionization processes may contribute to the Stream's $\mathrm{H} \alpha$ emission, and full treatments will require hydrodynamical simulations with radiative transport of internal and external radiation fields.

\subsection{The Magellanic Bridge}

The Magellanic Bridge of gas connecting the Magellanic Clouds was discovered in neutral hydrogen emission by Hindman et al. (1963), before the discovery of the Stream. Earlier work by Kerr et al. (1954) had shown the H I in the Clouds to be more extended than their stars, but had not detected the diffuse bridge in between the two galaxies. The Bridge has historically been treated as a different object than the Stream, and this distinction makes sense for a number of reasons: the two are spatially separate on the sky, a stellar population exists in the Bridge (Irwin et al. 1990, Demers \& Irwin 1991, Harris 2007, Bagheri et al. 2013, Noël et al. 2013, Skowron et al. 2014) but has not been found in the Stream (Recillas-Cruz 1982, Brück \& Hawkins 1983, Guhathakurta \& Reitzel 1998, though see Belokurov \& Koposov 2015), and the two structures are likely to have been formed at different times (Besla et al. 2012). However, given the recent progress in understanding the dynamics of the entire Magellanic System (Section 5), the Bridge and Stream can also be seen as separate components of a larger structure.

Like the Stream, the (gaseous) Bridge is well characterized. It has an H I gas mass of $1.84 \cdot 10^{8} \mathrm{M}_{\odot}$ (for $d=55 \mathrm{kpc}$; Brüns et al. 2005). Profile analyses show that the Bridge contains two principal components, at +214 and $+234 \mathrm{~km} / \mathrm{s}$ (McGee \& Newton 1986), with other components also present that can be connected to the SMC and LMC. Metallicity measurements based on UV absorption-line studies of two embedded hot stars (DI 1388 and DGIK 975) find abundances of $[\mathrm{Z} / \mathrm{H}]=-1.02 \pm 0.07$ and $-1.7<[\mathrm{Z} / \mathrm{H}]<-0.9$ (Lehner 2002; Lehner et al. 2008). Along a line-of-sight toward a QSO lying behind the Bridge, Misawa et al. (2009) find $-1.0<[\mathrm{Z} / \mathrm{H}]<-0.5$. The fact that all these values are close to the (current-day) SMC metallicity and not the LMC metallicity suggests that the Bridge was formed from material formerly in the SMC. The column densities of H I in the Bridge are high enough that Ca II absorption, which typically traces dense neutral gas, has been detected (Smoker et al. 2000, 2005). In the models of Besla et al. (2012), one potential scenario for Bridge formation was a direct collision between the SMC and LMC $\sim 100-300 \mathrm{Myr}$ ago. These models predict a metallicity gradient along the Bridge (increasing toward the LMC) owing to a contribution from LMC gas. This prediction could be tested by future observations.

Warm ionized gas in the Bridge was surveyed in $\mathrm{H} \alpha$ by Barger et al. (2013) using the Wisconsin $\mathrm{H} \alpha$ Mapper (WHAM) telescope (see Figure 3. They found a warm H II mass of $(0.7-1.7) \cdot 10^{8} \mathrm{M}_{\odot}$ in a region where $\mathrm{M}(\mathrm{H} \mathrm{I})=3.3 \cdot 10^{8} \mathrm{M}_{\odot}$. Their analysis shows that ionizing radiation from the extragalactic background and the MW is insufficient to explain the observed $\mathrm{H} \alpha$ flux; under a pure photo-ionization model (with no shock ionization of $\mathrm{H} \alpha$ ), they use the observed $\mathrm{H} \alpha$ intensities to derive an average escape fraction of ionizing photons of $<4.0 \%$ for the LMC and $<5.5 \%$ for the SMC. Detections of cool H I emission (Kobulnicky \& Dickey 1999), $\mathrm{H}_{2}$ absorption (Lehner 2002), and CO emission (Muller et al. 2003, Mizuno et al. 2006) show that the Bridge (like the Stream) is a multi-phase structure.

In their discovery of a stellar population in the Bridge, Irwin et al. (1990) found blue (young) main sequence stars between the SMC wing and the Western halo of the LMC. This young Bridge has been shown by Skowron et al. (2014) to form a continuous connection between the two Clouds. In addition, intermediate-age and old stellar populations exist 

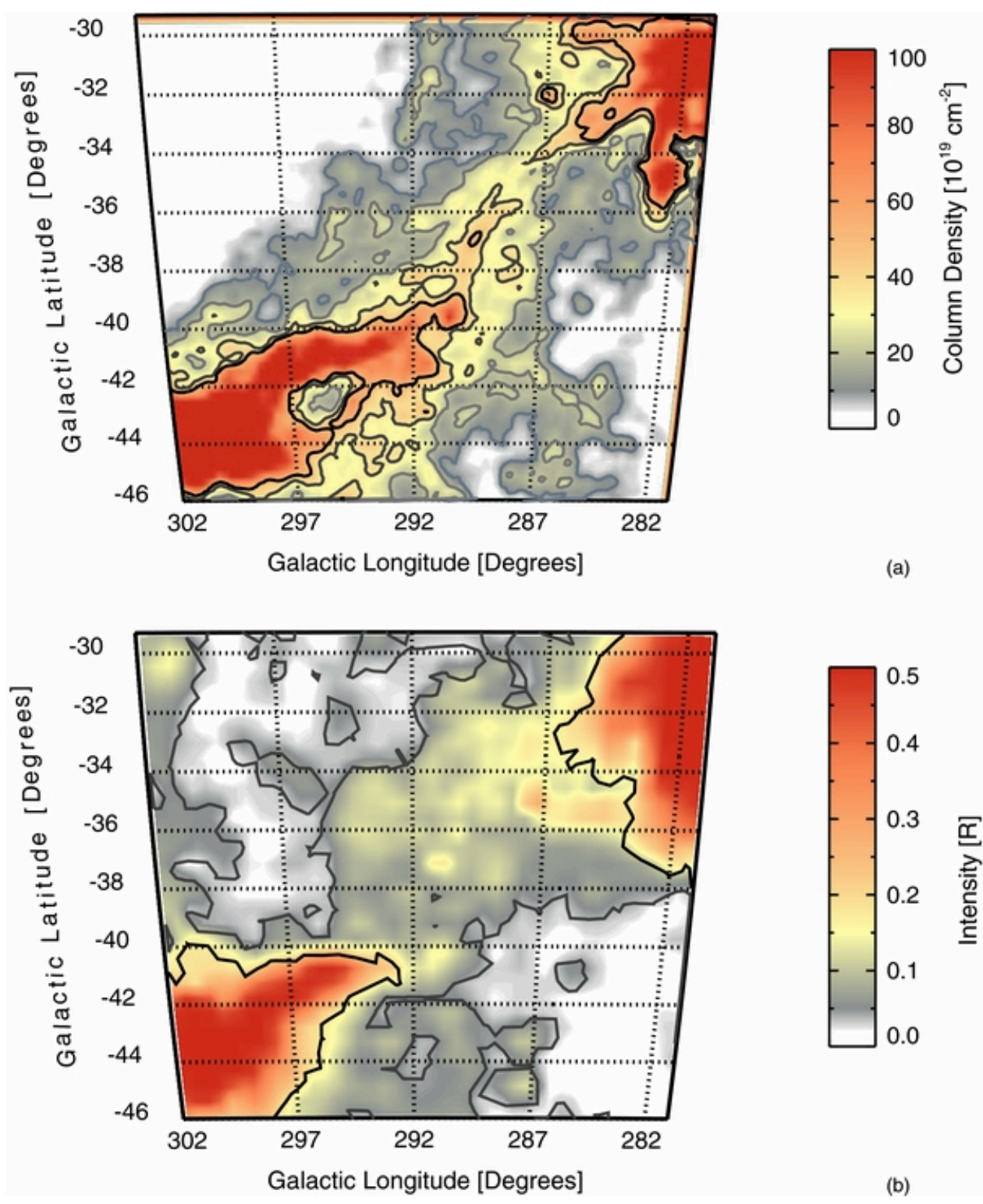

Figure 3

The Magellanic Bridge in H I (top) and $\mathrm{H} \alpha$ (bottom), using observations from GASS and WHAM, respectively (Barger et al. 2013). The SMC tail is at the lower left and the LMC is at the upper right. The GASS data are described in McClure-Griffiths et al. (2009).

(Bagheri et al. 2013; Noël et al. 2013). The origin models for the Magellanic System discussed in Section 3 need to account for this basic observation that the Bridge contains stellar populations whereas the Stream does not (at detectable surface density). 


\subsection{The Leading Arm}

The Leading Arm is the counterpart to the Stream on the opposite side of the Magellanic Clouds. Parts of the Leading Arm are visible in the original H I data presented by Mathewson et al. (1974), but its physical connection to the Clouds was not established until the work of Putman et al. (1998). The study of Lu et al. (1998), who measured a sulfur abundance of $(\mathrm{S} / \mathrm{H})=0.25 \pm 0.07$ solar (similar to the SMC metallicity) in the Leading Arm toward the AGN NGC 3783, was also key in demonstrating the Magellanic origin of the Leading Arm. This remains the best metallicity measurement in the Leading Arm to date.

The Leading Arm extends $\approx 60^{\circ}$ from the Clouds across the Galactic plane into the northern hemisphere where it disintegrates into a large number of small cloudlets (see Figure 1). For et al. (2014) and Venzmer et al. (2012) recently demonstrated that four principal Leading Arm sub-structures exist. The fact that the Leading Arm "leads" the orbits of the Magellanic Clouds in their motion around the Galaxy is an important constraint on its origin, since only tidal forces (and not ram-pressure stripping) are able to explain the presence of gas ahead of the Clouds' trajectory.

The Leading Arm deviates from the great circle defined by the Stream by $\approx 60^{\circ}$ (Putman et al. 1998). It has an $\mathrm{H} \mathrm{I}$ mass of $3 \cdot 0 \cdot 10^{7} \mathrm{M}_{\odot}$ (Brüns et al. 2005) and shows high positive velocities in the LSR frame (from $\approx+180 \mathrm{~km} / \mathrm{s}$ to $+270 \mathrm{~km} / \mathrm{s}$ ). Unlike the Stream, the Leading Arm has a fairly constant column density profile along its length (Nidever et al. 2010). Venzmer et al. (2012) have analyzed the role of drag forces in creating the Leading Arm structures seen in the GASS H I data. They find an inverse correlation between Galactic Standard of Rest velocities and Magellanic Longitudes (so the gas farthest from the Clouds is moving slower), and that around one quarter of Leading Arm clouds show head-tail morphologies that indicate an interaction with an external medium (see also McClure-Griffiths et al. 2008).

Casetti-Dinescu et al. (2014) recently detected 19 young massive stars in the Leading Arm (with ages $\approx 50-200 \mathrm{Myr}$ ), providing evidence for in-situ star formation, since there has not been enough time for O-stars stripped from the Clouds to reach their current location before evolving off the main sequence (an LMC star would have had to travel at $\approx 10^{4} \mathrm{~km} / \mathrm{s}$, which is clearly unrealistic). This shows that dense, star-forming molecular gas exists within the Leading Arm. Casetti-Dinescu et al. argue that the star formation may be triggered by the interaction with the Galactic corona. Such triggered star formation has not been seen in the Stream, perhaps because it is further away and interacting with a more tenuous halo medium. The evidence for triggered star formation in the Leading Arm is consistent with the high-level of disintegration and sub-structure observed in the Leading Arm (Putman et al. 1998, Venzmer et al. 2012).

\subsection{Total gas mass and inflow rate onto the Galactic halo}

The neutral gas mass of the Stream and the other components of the Magellanic System is well established from $21 \mathrm{~cm}$ surveys (Brüns et al. 2005). The four dense concentration MS I-IV account for $M(\mathrm{H} \mathrm{I})=1.25 \cdot 10^{8} \mathrm{M}_{\odot}$ assuming $d=55 \mathrm{kpc}$, the mean of the SMC and LMC distance, although this is likely a lower limit on the Stream's mass, since models find it could extend to $d=100 \mathrm{kpc}$ or beyond (Besla et al. 2010, 2012). A further $M(\mathrm{H} \mathrm{I})=1.49 \cdot 10^{8} \mathrm{M}_{\odot}$ exists in the "Interface region" defined by Brüns et al between the Magellanic Clouds and the rest of the Stream. In this review we treat the Interface region as part of the Stream, since the filaments of the Stream can be traced through it back to the Clouds, and so it is 
clearly part of the same structure. The Bridge accounts for $1.84 \cdot 10^{8} \mathrm{M}_{\odot}$ and the Leading Arm $3.0 \cdot 10^{7} \mathrm{M}_{\odot}$ of H I (Brüns et al. 2005). Together, the H I in the Magellanic System (not including the H I within the LMC and SMC themselves) amounts to $4.87 \cdot 10^{8} \mathrm{M}_{\odot}$, comparable to the H I mass within each of the LMC and SMC (see Table 1).

Determining the ionized gas mass is more challenging, since one must use metal lines as proxies for the ionized hydrogen atoms and make corrections for ionization and metallicity, which are both non-trivial. Furthermore, the metal lines are measured in pencil-beam directions and the covering fraction of ionized gas on the sky is larger than that of neutral gas, so a correction must be made for the cross-section. Fox et al. (2014) constrained the Stream's gas mass using photoionization models based on the Si II and Si III column densities measured in 19 extragalactic directions together with calculations of the radiation field emerging from the MW and the Magellanic Clouds. They report a total gas mass for the Magellanic System (Stream, Bridge, and Leading Arm) of $\approx 2.0 \cdot 10^{9} \mathrm{M}_{\odot}$, of which $\approx 25 \%$ is in neutral gas and $\approx 75 \%$ is in ionized gas (including warm ionized and highly ionized components). Given that the LMC contains $4.0 \cdot 10^{8} \mathrm{M}_{\odot}$ of interstellar H I and the SMC contains $4.4 \cdot 10^{8} \mathrm{M}_{\odot}$ at the current time (Staveley-Smith et al. 1997, Brüns et al. 2005), and that ionized gas is not thought to be a dominant contributor to the Clouds' global ISM mass budgets (most $\mathrm{H} \alpha$ emission in the LMC and SMC comes from supershells, not diffuse gas; Kennicutt et al. 1995), this leads to the conclusion that the Stream, Bridge, and Leading Arm together contain over twice as much gas as the current-day Magellanic Clouds. That is, if all the observed Magellanic gas (neutral and ionized) used to exist in the Clouds, then most of the original gas content of the Magellanic Clouds has been stripped. Thus while the Magellanic Clouds are often referred to as "gas-rich" satellites, we are observing them at a time when they may have lost most of their initial gas. As a note of caution, some of the ionized gas in the Stream may trace condensing hot material from the surrounding medium (Sect. 4), in which case the gas did not all originate from within the Clouds.

Dividing the Stream's gas mass by its inflow time onto the MW gives its (time-averaged) inflow rate. The inflow time is $\sim 0.5-1.0$ Gyr for $d=50-100 \mathrm{kpc}$ and its average Galactocentric inflow velocity of $100 \mathrm{~km} / \mathrm{s}$ (Mathewson et al. 1977, Mathewson 1985). This gives a mass inflow rate of $\sim 4-7 \mathrm{M}_{\odot} \mathrm{yr}^{-1}$ (Fox et al. 2014). This is larger than the inflow rate represented by all other Galactic HVCs, which is in the range $0.08-1.4 \mathrm{M}_{\odot} \mathrm{yr}^{-1}$ depending on assumptions about distance and ionization (Shull et al. 2009, Lehner \& Howk 2011, Putman et al. 2012). It is also larger than the current Galactic star formation rate, which is in the range $\approx 1-2 \mathrm{M}_{\odot} \mathrm{yr}^{-1}$ (Chomiuk \& Povich 2011, Robitaille \& Whitney 2010). Therefore the Stream is bringing in fuel at a rate sufficient to elevate the future Galactic star formation rate, and at a rate higher than that of any other observable infalling fuel source. The key question is whether the Stream will survive to reach the disk, evaporate into the hot halo, or even seed the cooling of hot halo gas and accrete more mass as it comes in. We will return to this question in Section 4

\section{ORIGIN OF THE MAGELLANIC STREAM}

The Stream has historically been explained as the outcome of two competing scenarios:

The Tidal Model. In this scenario the Stream is the outcome either of the tidal interactions between the LMC and the MW, as first suggested by Fujimoto \& Sofue (1976) and Lin \& Lynden-Bell (1977), or as material pulled out from the SMC by the tidal force 
exerted by the LMC and controlled by gravitational force of the MW, a model that traces back to Murai \& Fujimoto (1980) and more recently to Guglielmo et al. (2014). The tidal model envisions that a close encounter between the Magellanic Clouds occurred when they were separated by a distance of $2-3 \mathrm{kpc}$, with the dwarfs assumed to be a binary pair on short periodic orbits $(\sim 2$ Gyr $)$ around the MW. Several observations supported the tidal origin of the Stream, including the presence of the Bridge of gas and stars and the discovery of the Leading Arm.

The Drag Model. In this scenario the Stream is created by ram-pressure stripping as the Clouds pass through an external medium, which could be either the tenuous coronal gas in the Galactic halo (Meurer et al. 1985; Moore \& Davis 1994) or alternatively the denser gas of the Galactic disk. The latter would apply if the Clouds crossed the outer disk at some point in their past orbit. Later studies of these effects showed that a combination of tidal and hydrodynamical interactions can produce a close approximation of the Stream under the assumption that the Clouds had multiple close passages with our Galaxy (Gardiner \& Noguchi 1996; Mastropietro et al. 2005; Connors et al. 2006).

However, recent HST proper-motion measurements of the Clouds and developments in current models of galaxy formation have provided crucial new information regarding the Stream's history and origin. Because the new measurements suggest that the Clouds have either completed one orbit around the MW or may even be at first perigalacticon, a revised interpretation of the origin of the Stream may be required. Numerous observed features of the Stream, Bridge, and Leading Arm need to be accounted for by origin models and simulations.

- The spatial extension of the Stream and the Leading Arm, which together stretch to over $200^{\circ}$ on the sky, possibly crossing the Galactic disk in two locations, at the Leading Arm and at the Stream tip.

- The Stream presents a filamentary structure (Wakker 2001; Putman et al. 2003b) with at least two main filaments (Nidever et al. 2008), one with metallicity consistent with the LMC, and one more close in metallicity to the SMC (Fox et al. 2013, Richter et al. 2013).

- A dominant fraction of the Stream's mass is in the form of ionized gas (Fox et al. 2014).

- There is lack of a stellar counterpart in the Stream, despite the plentiful amount of gas in neutral and ionized form. This absence of stars in the Stream prevents distance measurements. However, recently 19 young stars in situ have been discovered in the Leading Arm, providing an approximate estimate to that region of $\sim 21 \mathrm{kpc}$ (CasettiDinescu et al. 2014).

It has been argued that the Stream is a young feature (1-2 Gyr; Besla et al. 2007), which provides a constraint on origin models. The arguments for a young Stream are twofold, but have attached caveats. First, simulations of the survivability of high-velocity clouds moving in a hot external medium (Heitsch \& Putman 2009; Joung et al. 2012) find that clouds evaporate on timescales of hundreds of Myr to $\sim 1$ Gyr. However, the cloud lifetime depends on the HVC mass: these simulations only considered HVCs with H I masses $<10^{4.5} \mathrm{M}_{\odot}$, whereas the largest Stream clumps are more massive (and longer lasting). Second, the Stream exhibits high $\mathrm{H} \alpha$ emission (see Section 2.2.4), which may indicate that its gas is being ablated away on a 100-200 Myr timescale (Bland-Hawthorn et al. 2007). However, 
the origin of the $\mathrm{H} \alpha$ emission is unclear: it may be shock-ionized and/or photoionized, so the $\mathrm{H} \alpha$ intensity does not provide a clean diagnostic on age. Therefore, there are no clear observational constraints on the Stream's age.

Despite the observational constraints listed above and numerous theoretical efforts and observational constraints, the origin of the Stream and Leading Arm remain uncertain. Recent $N$-body and hydrodynamic simulations (Besla et al. 2010, 2012; Diaz \& Bekki 2011b, 2012) have questioned the standard picture of Stream formation in which the Clouds traveled on a quasi-periodic orbit around the MW. These new models posit that the origin of the Stream was caused by the mutual tidal interaction between the Clouds before they were accreted by the MW (Besla et al. 2010; 2012) and on a bound orbit around the MW (Diaz \& Bekki 2011b,2012). The Galactic potential governs the orbits of our neighboring dwarfs and therefore causes the trail of the Stream to be amplified across the sky once the pair of dwarf galaxies fell in. The recent proper-motion estimates of the Clouds are consistent with one or two passages of the Clouds around the MW - a third passage is less likely - making it difficult to discriminate among these tidal scenarios.

\subsection{First-passage (unbound) scenarios}

In the first-passage scenario the LMC and SMC are just past their first pericentric passage (Besla et al. 2007). In this model the LMC first entered the virial radius of the MW within the past 1-4 Gyr and has not yet completed an orbit. This model was updated by Besla et al. (2012) to explore the morphology of the Stream produced from a head-on collision between the Clouds, specifically by the SMC moving in a highly eccentric orbit around the LMC, far from the MW potential. Highly eccentric orbits are cosmologically motivated as suggested by studies of satellite galaxies in current models of galaxy formation, but were never assumed in early studies on the formation of the Stream. While the high eccentricity of the orbit prevents the Clouds from merging in these new models, it requires the SMC to spend most of its time at apocenter (at $\sim 80-100 \mathrm{kpc}$ away from the LMC). At the first collision with the LMC the Stream forms as material tidally removed from the SMC, a process that does not require any interaction with the MW potential (and this is consistent with the Stream's low metallicity). The Clouds are assumed to be an interacting pair for a significant fraction of the Hubble time, and only recently fell into the MW potential (about 2 Gyr ago).

There is compelling evidence that the LMC and SMC had a recent and close encounter. First, the Magellanic Bridge of gas and stars appears to be a tidal feature (see e.g. Besla et al. 2012). Second, the LMC and SMC proper motions imply at least one collision within the past $500 \mathrm{Myr}$, as a direct result of the relative orientation of their three-dimensional velocity vectors (Kallivayalil et al. 2013). Third, the distribution of OB stars in the MCs and near the Bridge is also consistent with a recent exchange of material, 200 Myr ago (Casetti-Dinescu et al. 2013).

This picture is plausible within the current $\Lambda \mathrm{CDM}$ paradigm where halos at all scales build up their mass hierarchically. Interacting dwarf galaxies are therefore cosmologically expected both in isolation and on their orbits around more massive galaxies. When two disky galaxies pass by one another, in this case the SMC and LMC disks, their mutual gravitational tidal forces distort the disks of stars and gas resulting in the formation of tails and bridges of material (Toomre \& Toomre 1972). It has been shown that the efficiency of the process depends particularly on the inclinations of the disks relative to the orbit plane. 


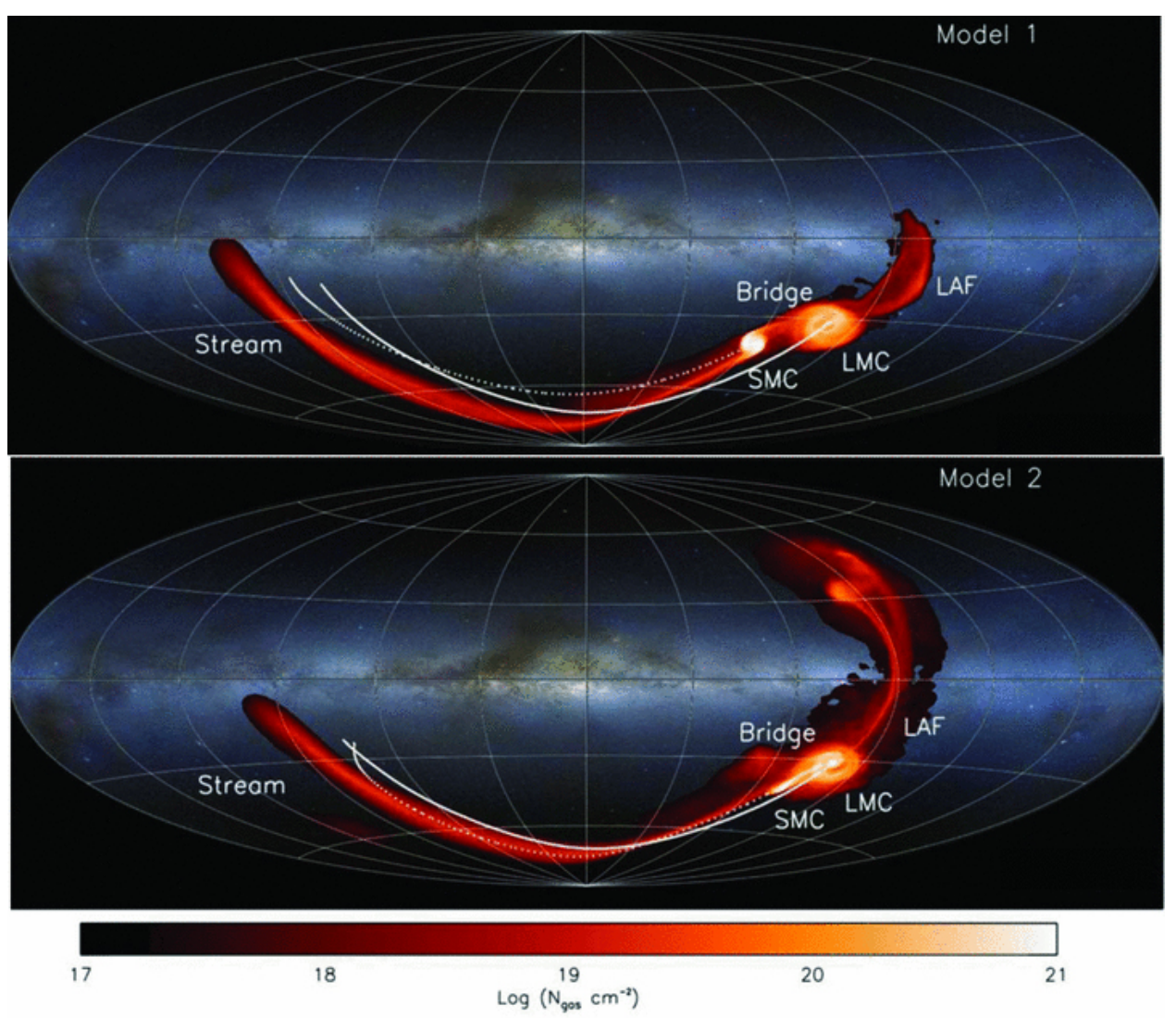

Figure 4

Gas projection in the simulated Magellanic System (red scale) plotted over an optical image of the MW. The models reproduce the length of the Stream and Leading Arm, as well as the Bridge connecting the Clouds. In the top panel (Model 1), the SMC has completed two passages around the LMC. In the lower panel (Model 2), it has completed three. Credit: Besla et al. (2012), with background image from Mellinger (2009).

Therefore, the mechanism is more efficient for co-planar and prograde encounters than for retrograde ones (Toomre \& Toomre 1972; D'Onghia et al. 2010; Łokas et al. 2015).

The Besla et al. (2012) first-passage models reproduce many observed features of the Stream (see Figure 4) in particular:

1) The location of the Stream across the sky is well approximated;

2) The resulting line-of-sight velocities for the simulated Stream agree with the data;

3) The simulated $\mathrm{H}$ I column densities range from $10^{18}-10^{21} \mathrm{~cm}^{-2}$ in agreement with the data, although the exact column density gradient along the length of the Stream is not reproduced (see Figure 8 in Besla et al. 2012). The column density is inhomogeneous across the width of the simulated stream: the inclusion of a realistic interaction with the MW halo gas might help in reproducing the bifurcated, filamentary nature of the observed Stream;

4) The pronounced asymmetry between the trailing and leading components is matched. 
Indeed, the Leading Arm of the simulated Stream is much smaller than the trailing component, as observed. This arises because the leading tidal arm from the SMC falls toward the LMC, while the trailing component stretches out to increasing distance.

Some difficulties are still experienced in the first-passage scenario, that call for additional physics to be eventually included in the models. In particular, the H I column density is one tenth of that observed (Besla et al. 2012). The tidal model underestimates the Stream's gas mass by a factor of $\approx 10-100$ when accounting for the huge (but not precisely constrained) amount of ionized gas.

Another difficulty with the first passage model is that one filament of the Stream originates in the LMC (Nidever et al. 2008; Richter et al. 2013). The fact that the LMC filament traces back to the starburst region 30 Doradus strongly suggests that star formation is linked to its origin. This is difficult to reconcile with the tidal model where the Stream gas is formed purely by direct collision between the Clouds.

A recent study explored the implications of the ram-pressure stripping experienced by the LMC moving at high speed through the Galactic halo gas at its first passage with the MW, employing both analytic prescriptions and full 3-dimensional hydrodynamic simulations (Salem et al. 2015). While this study shows that it is unlikely that material ram-pressure stripped from the LMC can account for more than a tiny percentage of the total mass of the Magellanic Stream, it does show that gas located to the North-West of the LMC's disk is enriched in metallicity by the contribution of gas ram-pressure stripped by the LMC.

The position of the Leading Arm in the models is inconsistent with its observed location. There is some evidence that the Leading Arm has already reached the Galactic disk: McClure-Griffiths et al. (2008) report an interaction between the Leading Arm cloud HVC 306-2+230 and the disk, which gives a kinematic distance to this part of the Leading Arm as $\approx 21 \mathrm{kpc}$ from the Sun, consistent with the distance to the newly-discovered stars in the Leading Arm (Casetti-Dinescu et al. 2014). This distance is close to that predicted by the Connors et al. (2006) and Yoshizawa \& Noguchi (2003) models, that advocate for at least one past passage of the Clouds with the MW.

It should be stressed that the first-infall scenario naturally reproduces the Bridge, through a high-speed encounter with the SMC passing through the center of the LMC. However, one question that remains puzzling and needs clarification concerns the metallicity and age of the Bridge. The Bridge has a measured gas-phase metallicity of only 0.1 solar (Lehner et al. 2008, Misawa et al. 2009), lower than the current-day abundances of both Clouds, and its age might indicate an earlier formation than 200-300 Myr ago, when the models posit it was formed.

\subsection{Multiple-passage (bound) scenarios}

In multiple-passage models where the Clouds follow a bound orbit, there is a clear difference in the role played by the MW in the origin of the Stream. The key feature of these models is that there is time for tidal interactions to occur between the Clouds. If the Clouds are on a bound orbit, the MW seems to govern the binary action of the LMC-SMC pair, guiding them into a recently formed short-period orbit (Diaz \& Bekki 2011b).

The Diaz \& Bekki model posits that the LMC-SMC system only recently formed a binary pair, within the last $\sim 2$ Gyr. In this scenario, the LMC and SMC may have originally formed as independent satellites of the MW, separated by large distances (Bekki et al. 2004). Their 
orbital evolution through the Galactic halo gradually brought them closer together until the LMC was able to capture the SMC into its orbit and form a tightly bound binary pair. While it is not clear how likely such a satellite capture is in models of galaxy formation, a bound orbit with the Clouds currently being on their second perigalacticon is not ruled out by the current measurements of the proper-motion of the Clouds. Interestingly, the model seems to reproduce the on-sky bifurcation of the Stream's two filaments better than the first-passage model, suggesting that a bound association with the MW favors the formation of such bifurcation.

A study of the global SFH of the LMC suggests that the LMC and SMC might have had coupled episodes of star formation, one occurring $\sim 2$ Gyr ago and one $\sim 500 \mathrm{Myr}$ ago (Harris \& Zaritsky 2009). It is unclear whether the timings of the two close LMC-SMC interactions in the first-passage and second-passage models are consistent with these burst epochs. The central theme of these tidal scenarios where the LMC and SMC are a strongly interacting pair is that the Stream originates from material pulled out of the Clouds. Because the gravitational field of the LMC will act in the same manner on gas and stars, we expect a tail of stars pulled out from the SMC into the Stream, especially in the case of a head-on collision. Unfortunately, this stream of stars still awaits discovery; searches for a stellar Stream have been unsuccessful (Recillas-Cruz 1982, Brück \& Hawkins 1983, Guhathakurta \& Reitzel 1998), although on the opposite (Eastern) side of LMC, a (possibly unrelated) stellar stream $10 \mathrm{kpc}$ long has recently been reported by Mackey et al. (2015; see also Belokurov \& Koposov 2015). While the 19 recently-discovered young stars in the Leading Arm (Casetti-Dinescu et al. 2014) appear to have formed in-situ, it is unclear whether this star formation was triggered by the interaction with the Galactic disk or halo. Future metallicity measurements of the Leading Arm gas near these stars will give insights on their origin.

\subsection{The role of ram-pressure stripping}

A pure ram-pressure-stripping origin for the Stream has been explored by many authors (Meurer et al. 1985, Moore \& Davis 1994, Heller \& Rohlfs 1994, Murali 2000, Mastropietro et al. 2005, Diaz \& Bekki 2011a). Such models naturally explain the lack of stars in the trailing Stream, but do not explain the existence of the Magellanic Bridge connecting the LMC and SMC or the origin of the Leading Arm (which leads the entire system). It is thus clear that tidal interactions between the Clouds must have played a role in the formation of the Magellanic System.

However, the detailed morphology of the Stream and the Leading Arm prove the complexity of the Magellanic System. Several simultaneous physical processes are needed to describe it. Recently, the Parkes Galactic All-Sky Survey (GASS; McClure- Griffiths et al. 2009) was used to identify the locations where the filaments cross each other and to explore the twisting filamentary system at higher resolution (Hammer et al. 2015). In an attempt to reproduce these two filamentary structures using hydrodynamic simulations, this study assumes that the MCs were following parallel orbits along the observed Stream. Thus, the Stream from the South Galactic Pole to the current location of the Clouds would be generated by a direct collision between the Clouds and the interaction with the MW hot gas. Leaving aside the question of how the Clouds are accreted into the Galactic halo, the model shows that the pair started to respond to the MW hot halo gas at a large distance from the MW and then had a mutual collision with a pericenter of $\approx 3 \mathrm{kpc} \approx 250 \mathrm{Myr}$ ago. The 


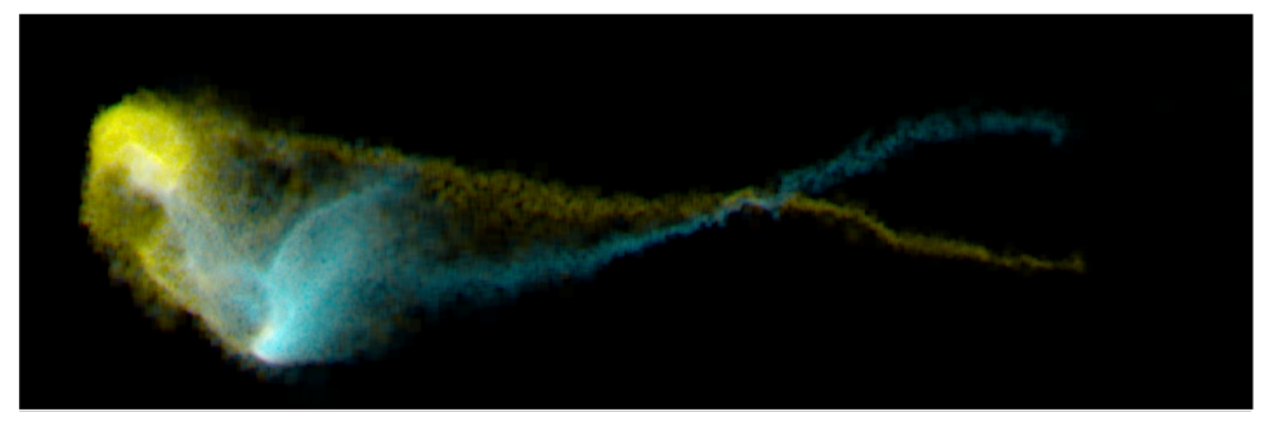

Figure 5

Simulation of the Stream that reproduces its spatial extent and filamentary nature. In this model the Magellanic Clouds collided $\approx 200-300 \mathrm{Myr}$ ago and the two filaments of the Stream are formed by the ram pressure exerted by a hot MW corona with a gas density $\approx 10^{-4} \mathrm{~cm}^{-3}$. Gas from the LMC is shown in yellow; gas from the SMC is shown in blue. Credit: Hammer et al. (2015).

outcome is two prominent ram-pressure tails trailing behind each Magellanic Cloud. The assumption of the Clouds traveling on parallel orbits and their recent collision produces the twin, intertwined filaments, as observed. The recent collision also produces a Magellanic Bridge. The resulting Stream from these models is shown in Figure 5 It would be interesting to assess the likelihood of such a orbital configuration for the Clouds in cosmological simulations.

\subsection{Other scenarios}

An alternative to the canonical tidal and ram-pressure scenarios envisions a primordial origin for the Stream (Peebles \& Tully 2013). According to this model, the Stream originated from tidal interactions at high redshift between the young LMC and the MW, which was its nearest massive neighbor at the time. This picture traces back to the model introduced by Fujimoto \& Sofue (1976) and Lin \& Lynden-Bell (1977), but applied at high redshift under cosmological initial conditions and solving the equation of motion for the Local Group. A first test of the viability of this model would be to check whether a primordial scenario can account for the Stream's length, width, and later interactions with the SMC, that we know at this point to be responsible of the Bridge.

It is worth mentioning that if the Clouds fell into the MW halo as part of a Magellanic Group (see Sect. 5.6) then they would naturally have had high-speed encounters in the past and the tidal processes invoked to account for the origin of the Stream would still apply, but it would be interesting to check with hydrodynamic simulations.

\section{FATE OF THE MAGELLANIC STREAM}

The Stream is clearly a substantial gas reservoir, with a mass-flow rate high enough to potentially elevate the future star formation rate of the MW (Sect. 2.5. However, one of the biggest open questions on the Stream is whether it will survive its inflow passage and reach the Galactic disk. The hydrodynamic interaction with the hot Galactic corona may dissipate the Stream and replenish the corona with new material. Several lines of evidence indicate that such an interaction is already underway. 
First, there is clear evidence for small-scale structure in H I, in the form of fragmentation and cloudlets observed in many locations around the main filaments of the Stream, as if the Stream is disintegrating as it plunges into the halo (Mirabel et al. 1979, Mirabel 1981, Putman et al. 1998, 2003b, Wakker et al. 2002, Stanimirović et al. 2002, 2008, Westmeier \& Koribalski 2008, For et al. 2014). Furthermore, Putman et al. (2003b) and For et al. (2014) found many of these clouds show head-tail cometary morphologies, with a dense core and a diffuse tail. These morphologies are indicative of cloud/corona interactions (Brüns et al. 2000, Quilis \& Moore 2001, Peek et al. 2007), in which the head indicates the direction of the cloud's motion and the tail contains material swept into the cloud's wake.

Second, UV absorption-line studies have shown that the Stream contains a high-ion phase traced by the Si IV, C IV, and O VI doublets (Sembach et al. 2003, Fox et al. 2005, 2010, 2014, Richter et al. 2013, Kumari et al. 2015), as discussed in Section 3.3. This phase appears to be collisionally ionized at a characteristic temperatures of a few times $10^{5} \mathrm{~K}$ and will arise at the boundary layers between the cool Stream and the hot corona. The high ions can be thought of as a signature of this interaction.

Third, hydrodynamical simulations have explored the lifetime of cool gas clouds against disruptive encounters with the coronal gas (notably Kelvin-Helmholtz instabilities), and find that for mass ranges appropriate for HVCs, the timescales are relatively short. Heitsch \& Putman (2009) found that for HVCs with H I masses $<10^{4.5} \mathrm{M}_{\odot}$ the evaporation timescales are $<100$ Myr. The less massive the cloud (or cloudlet), the shorter the disruption time. Similar disruption timescales are found by Bland-Hawthorn et al. 2007 and Joung et al. 2012. Magnetic fields, if present, may stabilize the clouds and increase their lifetime against instability.

Ultimately, whether the Stream survives its inflow passage to the MW depends on two timescales. If the gas disruption time is less than the inflow time $(\sim 0.5-1.0 \mathrm{Gyr})$, the Stream will not survive. However, the appropriate disruption timescale to adopt is not straightforward, because of the fragmentation of the Stream into structures on a range of spatial scales. The larger, coherent structures such as the principal filaments are more likely to survive, but the smaller cloudlets $\left(<10^{4.5} \mathrm{M}_{\odot}\right)$ will not. Furthermore, the presence of a magnetic field, which has been detected at the $\sim 6 \mu \mathrm{G}$ level in the Leading Arm (McClureGriffiths et al. 2010), may stabilize cool clouds against evaporation.

This picture becomes more complicated once the condensation of coronal gas is considered. When a cool cloud interacts with the hot halo, the two phases mix at the cloud boundary, leading to the evaporation of cool gas and the condensation of hot gas. Depending on which process dominates, the mass of the cloud can either shrink or grow with time. These processes have been explored in the context of other HVCs in the Galactic halo (Marinacci et al. 2010, Fraternali et al. 2013, 2015; Marasco et al 2013). The balance of evaporation vs. condensation depend on the metallicity of the corona, since the condensation is driven by cooling, which scales with metallicity. It appears unlikely that condensation would dominate in a case with 0.1 solar metallicity, as is appropriate for most of the Stream, since the cooling rate is so low in this regime.

\section{KINEMATICS AND DYNAMICS OF THE MAGELLANIC SYSTEM}

\subsection{Kinematics of the LMC}

The dynamics, structure, and star formation history of the LMC have long been interpreted in the context of its proximity to both the MW and its dwarf-galaxy companion SMC. This 
has allowed for detailed and thorough analyses of its structure and kinematics, with much work in the last decade based on measuring the photometry and kinematics of large samples of individual stars, and deep star counts to measure the true extent of the LMC. Our knowledge of the internal kinematics of the LMC has improved over the last two decades, with different observational tracers covering different components of the Magellanic System. This includes kinematic measurements of the H I gas (Kim et al. 1998; Staveley-Smith et al. 2003), star clusters (Grocholski et al. 2006), and stars (Prevot et al. 1985; Massey \& Olsen 2003, Kunkel et al. 1997; van der Marel et al. 2002, Olsen \& Massey 2007; Olsen et al. 2011).

The rotation curves of galaxies are based on line-of-sight velocities and so are onedimensional. For most galaxies, proper-motion measurements are not possible within current observational capabilities, so three-dimensional kinematic information is unavailable, but the Magellanic Clouds are an exception. The recent LMC proper-motion measurements led to the first measurement of the large-scale rotation field in three dimensions (van der Marel \& Kallivayalil 2014). These measurements are fundamental to our understanding of the dynamics and mass of our largest dwarf companion. The proper-motion measurements imply an amplitude for the LMC rotation curve of $v_{0, P M}=76.1 \pm 7.6 \mathrm{~km} / \mathrm{s}$, obtained with a magnitude-limited sample of stars with mixed populations. This measurement falls in the middle between the measurements of the line-of-sight velocities of old and young stellar populations with velocities $v_{0, L O S}=55.2 \pm 10.3 \mathrm{~km} / \mathrm{s}$ and $v_{0, L O S}=89.3 \pm 18.8$ $\mathrm{km} / \mathrm{s}$, respectively (van der Marel \& Kallivayalil 2014). The major uncertainties on these measurements are due to the inclination of the LMC.

The rotation curve of the LMC peaks at $v_{c i r}=91.7 \pm 18.8 \mathrm{~km} / \mathrm{s}$, which implies an enclosed mass $\mathrm{M}_{\mathrm{LMC}}=(1.7 \pm 0.7) \cdot 10^{10} \mathrm{M}_{\odot}$ within a radius of $8.7 \mathrm{kpc}$ (see Table 1 ). The total dynamical mass of the LMC extends beyond this radius but is hard to determine. Given the fact that the LMC is in tidal interaction with the MW, its dark halo might be tidally truncated. An estimate of the inferred tidal radius is $22.3 \pm 5.2 \mathrm{kpc}$ (van der Marel \& Kallivayalil 2014).

Furthermore, through the study of the LMC proper motion rotation field it has been possible to address the location of the stellar dynamical center of the LMC, and whether it coincides with the H I dynamical center. Different measurements of the LMC's stellar center using different components or techniques are not spatially coincident, indicating that there is not a single well-defined center (van der Marel 2001; Cole et al. 2005). The proper-motion rotation fields recently inferred addressed this question. These measurements indicate that the dynamical center inferred by proper motion agrees with the $\mathrm{H}$ I dynamical center and the offset with the line-of-sight is greatly reduced. Uncertainties coming from the assumptions made in the model to infer the line-of-sight velocities may also introduce an offset between the dynamical center and the large line-of-sight velocity-field. In particular, the structure of the LMC turned out to be more complicated than a flat circular rotating disk (van der Marel \& Kallivayalil 2014).

An ongoing area of research related to the structure of the LMC concerns its stellar bar. Despite a wealth of data there is still great uncertainty concerning the bar's nature. The work of van der Marel (2001) indicates that the bar is off-centered from the dynamical center of the LMC and that it resides within a large stellar disk. Later work proposed that this feature might not be a bar, but instead a triaxial stellar bulge residing in a thick disk (Zaritsky 2004). Interestingly, the bar is not present in the comprehensive maps of the $\mathrm{H}$ I distribution and kinematics of the LMC (Kim et al. 1998, Staveley-Smith et al. 2003), 
despite the fact that the feature is clearly visible in the stellar distribution of the LMC. The OGLE III survey (Udalski et al. 2008) has been used to argue that the LMCs bar resides in the plane of the disk (Subramaniam \& Subramanian 2009). Earlier work described the bar as being an unvirialized structure that is offset from the rest of the disk as a result of the LMC's interaction with the SMC (Zhao \& Evans 2004). Recent numerical experiments of collisions between the SMC and LMC dwarf galaxies supported these findings (Besla et al. 2012, Yozin \& Bekki 2014). However, $N$-body simulations of interacting dwarf galaxies with properties similar to the Clouds show that the dynamical center and the bar center always coincide, but the photometric center is mismatched with the dynamical center due to tidally-induced distortions of the stellar disk (Pardy et al. 2015, in prep.). If this is the case it would be interesting to place the LMC dynamical center on the bar instead of the HI dynamical center. This would slightly change the north component of the proper motions: $\mu_{N}$, perhaps reducing the offset between the LMC's orbit and the position of the Stream.

\subsection{Kinematics of the SMC}

The intermediate-age and old stellar populations of the SMC, traced by carbon stars (e.g. Kunkel et al. 2000) and planetary nebulae (Dopita et al. 1985) show an absence of rotation. However, the young stellar population, traced by H I gas, shows a velocity gradient (Staveley-Smith et al. 1997).

The kinematics of the different components of the SMC are consistent with the picture of the SMC originally having been a disk-like dwarf galaxy before experiencing recent strong tidal interactions with the LMC. While there is no evidence of tell-tale tidal tails, the velocity field of the central region of the SMC is consistent with a recent tidal encounter with the LMC. When a spinning system like a disky dwarf galaxy encounters a more massive system like the LMC or the MW, it will lose mass by tidal stripping and will undergo a morphological transformation, with angular momentum being removed after the encounter (Mayer et al. 2001; D'Onghia et al. 2009). However, even after several encounters a velocity gradient is still expected to be present in the central regions of the SMC if gas and stars are rotationally supported, which seems to be consistent with current data. The SMC rotation curve inferred by $\mathrm{H}$ I data shows a rise up to a distance of $3 \mathrm{kpc}$ from the center and reaches a maximum of $60 \mathrm{~km} / \mathrm{s}$, corresponding to an enclosed total mass of $2.4 \cdot 10^{9} \mathrm{M}_{\odot}$, within that $3 \mathrm{kpc}$ radius (Stanimirović et al. 2004). Detailed three-dimensional propermotion measurements like the ones inferred for the LMC are needed to better constrain the properties of the SMC, especially in light of its role in the formation of the Stream.

\subsection{Proper motions and orbital history of the Magellanic Clouds}

The unusual situation of having a pair of Magellanic galaxies so close to a large spiral like the MW results in interactions in the LMC-SMC-MW system not seen in isolated Magellanic pairs. This situation is so uncommon that recent studies based on cosmological simulations and observations have concluded that less than $10 \%$ of MW-like galaxies are expected to host satellites with properties similar to the Clouds (Busha et al. 2010; Boylan-Kolchin et al. 2011, Tollerud et al. 2011).

A description of the global dynamics of the Magellanic System is needed before processes like star formation, chemical evolution, and kinematics in the LMC and SMC can be fully understood. Conversely, these processes can give information on parameters of the interactions. Thus the interacting Magellanic System is a key probe into many aspects of our 
Galaxy, from its mass to its halo environment, as well as being a doorway to understanding the formation and evolution of galaxies in general.

High-precision proper-motion measurements of LMC stars based on HST data taken in the last decade (see Section 5.4 Kallivayalil et el. 2006a,b, 2013; Piatek et al. 2008) indicate that the LMC has a higher tangential velocity than previously thought (the latest measurement is $v_{\text {tan }} \sim 314 \mathrm{~km} / \mathrm{s}$; Kallivayalil et al. 2013). A high tangential velocity for the LMC means that the centrifugal force is larger than the gravitational force acting on the LMC, thus the LMC is moving toward larger radii and so must be now at perigalacticon. The high tangential velocity raises questions as to whether the Clouds are actually bound to each other or even to the MW as a whole.

Early models assumed that the Clouds' orbits were slowly decaying into the MW halo potential by dynamical friction (Murai \& Fujimoto 1980, hereafter MF80; Davies \& Wright 1977; Lin \& Lynden-Bell 1977, 1982; Gardiner et al. 1994; Heller \& Rohlfs 1994; Moore \& Davis 1994; Lin et al. 1995; Gardiner \& Noguchi 1996; Bekki \& Chiba 2005, 2007; Yoshizawa \& Noguchi 2003; Connors et al. 2004, 2006; Mastropietro et al. 2005). Because of the large uncertainties in previous proper-motion measurements, the orbital parameters were chosen to best reproduce the properties of the Stream under the assumption that the LMC and SMC form a binary system that has been in a slowly decaying orbit around the MW for nearly a Hubble time.

In the next section, we discuss the proper-motion measurements of the Clouds based on the three-epoch HST data and discuss how these measurements affect the orbital history of the LMC and SMC and hence the origin of the Stream.

The proper motion is defined in the west $\left(\mu_{W}\right)$ and north $\left(\mu_{N}\right)$ directions as the variation in time of the right ascension $\alpha$ and declination $\delta$ in the sky:

$$
\mu_{W}=-(d \alpha / d t) \cos \delta, \quad \mu_{N}=-d \delta / d t
$$

The two components of the proper motion control the orbital parameters of the LMC. The combined west and north components determine the tangential velocity of the LMC and thereby sets the orbital period, number of pericentric passages, apogalactic distance, and the stability of the SMC-LMC binary system. The north component controls the location of the orbit when projected on the plane of the sky.

\subsection{Proper motions from the Hubble Space Telescope}

The advent of high-precision proper-motion measurements of the Clouds using HST data (Kallivayalil et al. 2006a, 2006b, 2013, hereafter K1, K2, K3; Piatek et al. 2008, hereafter P08) and a more physically motivated modeling of the Magellanic Clouds (Besla et al. 2007) have revolutionized our view of Magellanic dynamics. Following earlier results based on two epochs (K1, K2), a third epoch of HST data of ten QSOs behind the LMC and three behind the SMC has been recently analyzed (K3). The combined data give a 7-year baseline of proper-motion measurements for both Clouds. A comparison between the latest proper-motion measurements and the earlier measurements is given in Table 2 2 Table 3 lists the $3 \mathrm{D}$ velocity in the Galactocentric restframe of both the LMC and SMC, and the relative velocity of the $\mathrm{SMC}$ with respect to the LMC, as reported in $\mathrm{K} 3$.

The LMC proper-motion measurements obtained from three epochs of HST data (K3) provided the following results and implications:

- The three-dimensional speed of the LMC is lower (by $\sim 57 \mathrm{~km} / \mathrm{s}$ ) than measured from 
Table 2 LMC and SMC Proper Motion Measurements

\begin{tabular}{|c|c|c|c|c|c|}
\hline \multirow[t]{2}{*}{ Study Method } & \multicolumn{2}{|l|}{ LMC } & \multicolumn{2}{|l|}{ SMC } & \multirow[t]{2}{*}{ Refs. } \\
\hline & $\begin{array}{l}\mu_{W} \\
(\mathrm{mas} / \mathrm{yr})\end{array}$ & $\begin{array}{l}\mu_{N} \\
(\operatorname{mas} / \mathrm{yr})\end{array}$ & $\begin{array}{l}\mu_{W} \\
(\text { mas/yr) }\end{array}$ & $\begin{array}{l}\mu_{N} \\
(\text { mas/yr) }\end{array}$ & \\
\hline MS Model (G94) & -1.72 & 0.12 & $\ldots$ & $\ldots$ & $(1,2)$ \\
\hline MS Model (HR94) & -2.0 & 0.16 & $\ldots$ & $\ldots$ & $(3)$ \\
\hline Ground-based+Hipparcos ${ }^{a}$ & $-1.68 \pm 0.16$ & $0.34 \pm 0.16$ & $\ldots$ & $\ldots$ & (4) \\
\hline$H S T$ two-epoch $(\mathrm{K} 1, \mathrm{~K} 2)$ & $-2.03 \pm 0.08$ & $0.44 \pm 0.05$ & $-1.16 \pm 0.18$ & $-1.17 \pm 0.18$ & (5) \\
\hline$H S T$ two-epoch (P08) & $-1.56 \pm 0.036$ & $0.435 \pm 0.036$ & $-0.754 \pm 0.061$ & $-1.252 \pm 0.058$ & (6) \\
\hline $2.5 \mathrm{~m} \mathrm{du}$ Pont & $-1.72 \pm 0.13$ & $0.50 \pm 0.15$ & $-0.93 \pm 0.14$ & $-1.25 \pm 0.11$ & (7) \\
\hline Southern Proper Motion & $-1.89 \pm 0.27$ & $0.39 \pm 0.27$ & $-0.98 \pm 0.30$ & $-1.10 \pm 0.29$ & (8) \\
\hline$H S T$ three-epoch (K3) & $-1.910 \pm 0.02$ & $0.229 \pm 0.047$ & $-0.772 \pm 0.063$ & $-1.117 \pm 0.061$ & (9) \\
\hline
\end{tabular}

References: (1) Gardiner et al. 1994; (2) Gardiner \& Noguchi 1996; (3) Heller \& Rohlfs 1994; (4) van der

Marel et al. 2002; (5) Kallivayalil et al 2006a; 2006b; (6) Piatek et al. 2008; (7) Costa et al. 2011; (8)

Vieira et al. 2011; (9) Kallivayalil et al. 2013.

${ }^{a}$ Value weighted average of the ground-based and Hipparcos measurements by Kroupa et al. 1994; Jones

et al. 1994; Kroupa \& Bastian 1997; Pedreros et al. 2002; Drake et al. 2001.

the two-epoch measurements, owing to the decrease in the west component of the proper motion and the new measurement of the solar velocity. For a MW mass of $1 \cdot 10^{12} \mathrm{M}_{\odot}$ these measurements imply that the LMC might be on a parabolic orbit, with the LMC just past perigalacticon (K3). Alternatively, if the mass of the MW is higher at $\approx 2 \cdot 10^{12} \mathrm{M}_{\odot}$, the LMC will be on a more eccentric orbit with a period of $\approx 6 \mathrm{Gyr}$, and currently at its second pericentric passage (Besla et al. 2007, Piatek et al. 2008, Shattow \& Loeb 2009, K3).

- The current measurement of the north component of the proper motion $\mu_{N}=0.229 \pm$ 0.047 (Table 2 indicates that the LMC's orbit slightly deviates from the position of the Stream on the sky. This modest offset implies that the LMC orbit is not a close tracer of the Stream. The assumption that the Stream is aligned with the past orbit of the Clouds is one of the assertions of the tidal-stripping scenario for the Stream's origin. The SMC proper motion measurement suggest that in the past the SMC crossed the location of Stream, an orbital solution that agrees with the picture of the Stream being torn off the Clouds by their mutual tidal interactions (GN96; Růžička et al. 2009, 2010; Diaz \& Bekki 2011b; Besla et al. 2012).

- The observed relative velocity between the Clouds indicates that the radial component $v_{\text {rad }}$ of the LMC-SMC system is larger than the tangential component $v_{\text {tan }}$, suggesting that the Clouds are on an eccentric orbit.

The interpretation of the orbital history of the Clouds based on the proper-motion measurements crucially depends on the circular velocity inferred for our Galaxy and how it compares to the tangential velocity of the LMC.

An increase of a factor of two in the virial mass of the MW makes a difference (Piatek et al. 2008; Shattow \& Loeb 2009), which is certainly within the current uncertainty in virial mass estimates for the MW (e.g. Smith et al. 2007, Li \& White 2008, Reid et al. 2009). In fact the escape speed at $r=50 \mathrm{kpc}$ from a NFW halo (Navarro et al. 1997) with a virial mass ${ }^{2} \mathrm{M}_{200}=2 \cdot 10^{12} \mathrm{M}_{\odot}$ is of the order of $500 \mathrm{~km} / \mathrm{s}$, which would indicate that

\footnotetext{
${ }^{2}$ The virial mass, $M_{200}$ is defined as the mass contained within $r_{200}$, the radius of a sphere
} 


\begin{tabular}{cllllll} 
Table 3 & Three-epoch & \multicolumn{4}{c}{ HST Measurements: } & \multicolumn{3}{c}{ Galactocentric Velocities } \\
\hline Galaxy & $v_{X}$ & $v_{Y}$ & $v_{Z}$ & $v_{\text {tot }}$ & $v_{\text {rad }}$ & $\begin{array}{l}v_{\text {tan }} \\
(\mathrm{km} / \mathrm{s})\end{array}$ \\
& $(\mathrm{km} / \mathrm{s})$ & $(\mathrm{km} / \mathrm{s})$ & $(\mathrm{km} / \mathrm{s})$ & $(\mathrm{km} / \mathrm{s})$ & $(\mathrm{km} / \mathrm{s})$ \\
\hline LMC $^{*}$ & $-57 \pm 13$ & $-226 \pm 15$ & $221 \pm 19$ & $321 \pm 24$ & $64 \pm 7$ & $314 \pm 24$ \\
SMC $^{*}$ & $19 \pm 18$ & $-153 \pm 21$ & $153 \pm 17$ & $217 \pm 26$ & $-11 \pm 5$ & $217 \pm 26$ \\
SMC-LMC $^{*}$ & $76 \pm 22$ & $73 \pm 26$ & $-68 \pm 25$ & $128 \pm 32$ & $112 \pm 32$ & $61 \pm 16$
\end{tabular}

Credit: Kallivayalil et al. 2013

* Measurements based on the velocities used to correct for solar reflex motion: the improved McMillan

(2011) value of $v_{0}=239 \pm 5 \mathrm{~km} / \mathrm{s}$ and the solar peculiar velocity reported by Schönrich et al. (2010).

the Clouds are bound to the Galaxy despite their high speed (Sales et al. 2011).

Additional uncertainties in the measurements of the MW's circular velocity affect the measurement of the proper motions of the LMC, because this is measured relative to the Solar System that orbits the Galaxy. The rotational velocity of the Sun is therefore needed in order to transform to the Galactocentric frame (van der Marel et al. 2002). Although the recent $\sim 14 \pm 6 \%$ increase in the MW circular velocity relative to the International Astronomical Union (IAU) standard of $220 \mathrm{~km} / \mathrm{s}$ is included in the analysis of the proper-motion based on the third epoch of HST data (K3), the measurement of the solar proper motion is still debated.

\subsection{Orbital history of the LMC: parabolic or bound?}

Given the new Galactocentric velocities derived from the three-epoch HST data, the challenge is to determine whether the LMC is on a parabolic or on a bound orbit around the MW. This question depends on the modeling rather than the measurements of the velocities. Thus there are good reasons to believe that this debate will not be settled by further improving the precision of future measurements of proper motions. On the contrary, it depends crucially on the Galactic mass distribution, its total mass and virial radius, and how the Galactic potential evolves in time.

In the following we offer an example of the limitations of the models in trying to constrain the orbital history of the Clouds. The LMC orbit has been obtained by solving the differential equations of motion (see Murai \& Fujimoto 1980, Besla et al. 2007, Guglielmo et al. 2014):

$$
\ddot{\mathbf{r}}=\frac{\partial}{\partial \mathbf{r}} \Phi_{\mathrm{MW}}(|\mathbf{r}|)+\frac{\mathcal{F}_{d f}}{M_{\mathrm{LMC}}}
$$

Here $\mathrm{M}_{\mathrm{LMC}}$ is the mass of the LMC, $\mathbf{r}$ is its position vector, $\Phi_{\mathrm{MW}}$ is the Galactic potential, and $\mathcal{F}_{d f}$ is the dynamical friction term that assumes the form:

$$
\mathcal{F}_{d f}=-\frac{4 \pi G^{2} M_{\mathrm{LMC}}^{2} \ln (\Lambda) \rho(r)}{v^{2}}\left[\operatorname{erf}(\mathrm{X})-\frac{2 \mathrm{X}}{\sqrt{\pi}} \exp \left(-\mathrm{X}^{2}\right)\right] \frac{\mathbf{v}}{\mathrm{v}}
$$

where $\rho(r)$ is the density of the host halo at the Galactocentric distance of $50 \mathrm{kpc}$ (the distance of the LMC), $\ln \Lambda$ is the Coulomb logarithm, $v$ is orbital velocity of the LMC and $X=v / \sqrt{2} \sigma$. Here, $\sigma$ is the one-dimensional velocity dispersion of the dark matter halo.

of mean density 200 times the critical density for closure, $\rho_{\text {crit }}=3 \mathrm{H}^{2} / 8 \pi G$. This choice defines implicitly the halo virial radius $r_{200}$ and its virial velocity $V_{200}$. 

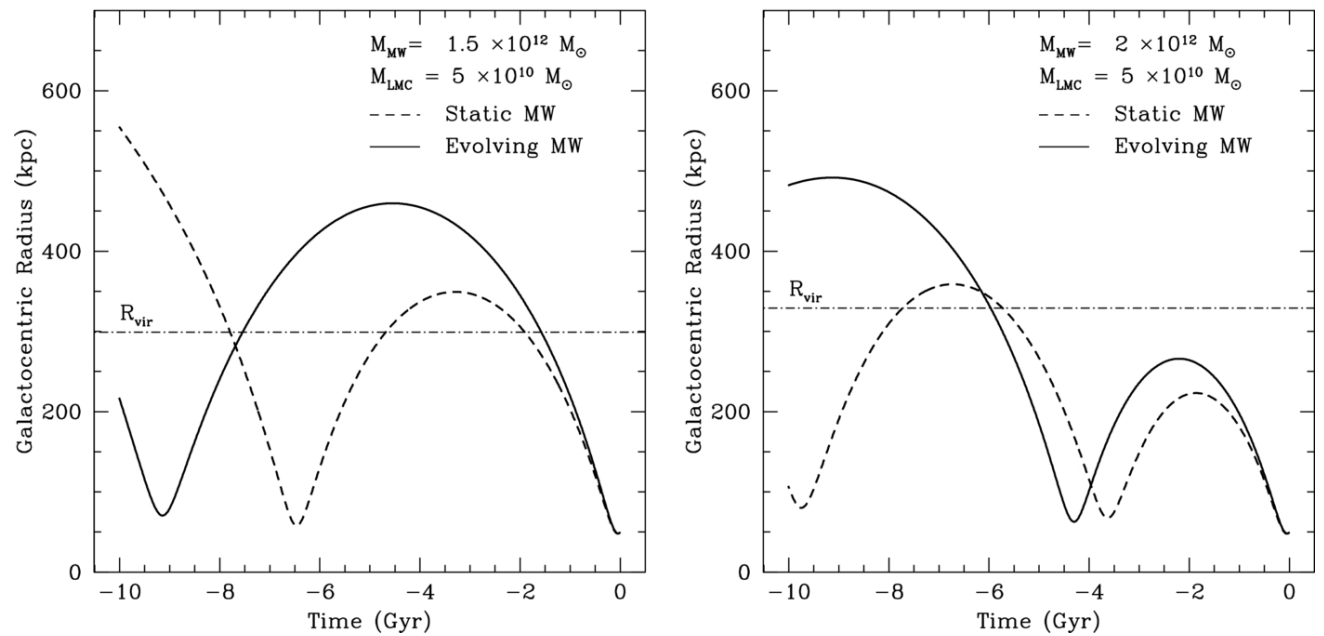

Figure 6

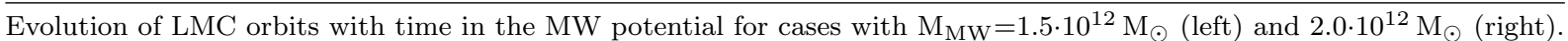
The mass of the LMC is assumed to be $\mathrm{M}_{\mathrm{LMC}}=5 \cdot 10^{12} \mathrm{M}_{\odot}$ in both models. In each case, the two lines show the cases of a static (fixed MW mass; dashed) and evolving (increasing MW mass; solid) Galactic potential. An evolving MW potential gives a longer orbit period for the LMC than the case of a fixed MW mass. Credit: Kallivayalil et al. (2013).

By imposing the current velocities and positions for the LMC, the differential equations of motion have been used to determine the position and velocities of the LMC at earlier times.

While this approach has been useful in early works that assumed that the Clouds' orbit were slowly decaying into the Galactic potential by dynamical friction, it relies on assumptions that require to be tested to make the orbital solution plausible. First, the formalism assumes that the LMC evolves in a rigid Galactic potential, which is probably not realistic. Second, the orbit of the LMC decays by dynamical friction and by tidal massloss. However, the dynamical friction term depends on the mass of the LMC which is tidally stripped by time, a situation that requires full $N$-body simulations to be captured.

Figure 6 shows that the LMC orbit period becomes longer if the Cloud feel the MW potential that evolves in time as compared to orbits computed in a static Galactic potential. Increasing the mass of MW models results in shorter LMC orbital periods. Gomez et al. (2015) argue that a massive LMC falling into the MW can perturb the Galactic potential. The result is a dynamical drag on the LMC motion that tends to shorten its orbit period and favor a scenario where the LMC is at currently at past second perigalacticon. In Figure 7 the orbits of the LMC (solid black lines) and SMC (red dashed lines) in the MW potential are displayed for two cases: first- and second passages. The first passage solution is obtained for a massive LMC falling into a massive MW halo, not accounting for the MW dynamical response to the massive LMC. When the dynamical drag is accounted for the LMC orbit period shortens, leading to a second pericentric passage. These orbital solutions (Figures 6 and 7 ) illustrate how the orbital history depends on the assumption made on modeling, rather than on the proper-motion measurements.

Another question related to the past orbital history of the Clouds concerns whether the LMC and SMC were and still are a bound pair. First-passage models where the Stream represents the debris of the mutual tidal interaction between the LMC/SMC before they 

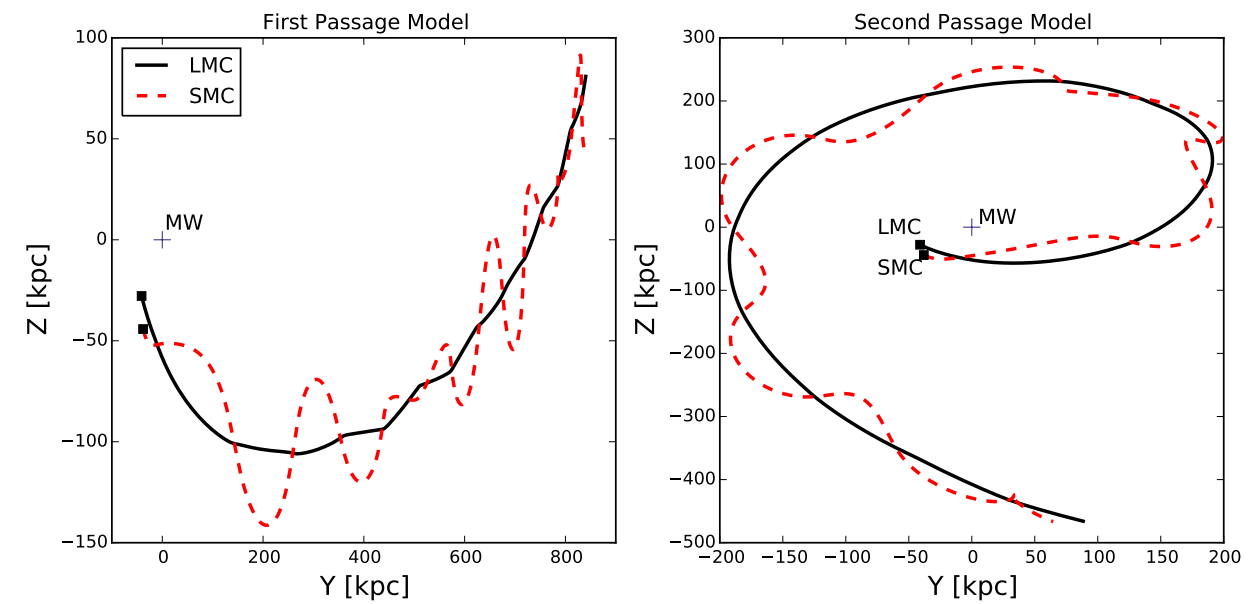

Figure 7

Two distinct orbital solutions for the LMC (black curve) and SMC (red dashed curve) in the MW potential, illustrating the first- (left) and second- (right) passage scenarios. The masses assumed for the MW and the LMC are: $\mathrm{M}_{\mathrm{MW}}=1.5 \cdot 10^{12} \mathrm{M}_{\odot}$ and $\mathrm{M}_{\mathrm{LMC}}=1.8 \cdot 10^{11} \mathrm{M}_{\odot}$. The effect of dynamical drag on the motion of LMC perturbing the MW is accounted for and results in a shorter orbital period for the LMC, favoring a double passage around the MW (right panel). Credit: this work.

fell into the MW require the Clouds to be bound at least for the last 4 Gyr and eventually have formed the Stream in the last 2 Gyr (Besla et al. 2012). Since the relative velocity between the Clouds is $\sim 130 \mathrm{~km} / \mathrm{s}$, LMC masses of the order of $10^{11} \mathrm{M}_{\odot}$ are needed in these models to explain how the LMC can have held on to the SMC. Thus these models require a low-mass MW $\left(\sim 10^{12} \mathrm{M}_{\odot}\right)$ and a massive $\mathrm{LMC}\left(\sim 10^{11} \mathrm{M}_{\odot}\right)$ to ensure that the Clouds are a long-term binary system.

In conclusion, our ability to properly model the orbital history of the Clouds depends on the ability to measure the potential of our Galaxy, e.g. by accurate measurements of the rotation curve, and on our understanding of the processes involved in galaxy formation, from the galaxy mass distribution, the satellite orbits, the environment, to gas dynamics. Thus, the progress of cosmological simulations of galaxy formation has the promise to better constrain the kinematics and dynamics of the Clouds and the Stream. Further progress in proper motion measurements, as will be possible with the Gaia mission, and, equally important, continued advances in the art of distance measurements, will further tighten the constraints, and may show us whether we really understand how dynamics is operating in our closest dwarf companions.

\subsection{The Magellanic Group}

The fact that the Clouds are so close to each other and embedded in the Magellanic Bridge of neutral hydrogen advocates for their physical connection. Using a projection of the bright dwarf galaxies of the MW onto a map of high-velocity hydrogen, Lynden-Bell (1976) suggested that, like the Magellanic Clouds, other near-neighboring dwarf galaxies were associated with the H I Stream. This was supported by the polar distribution of the 
brightest Galactic satellites, which seems to trace the orbital path of the Clouds. The number of dwarf galaxies in this association has increased with time and initially included the following candidates: the Magellanic Clouds, Ursa Minor, and Draco. Sculptor and Carina would also be added to the association of galaxies in the Magellanic Stream, though their potential membership is more tentative as their orientations are not along the Stream (Lynden-Bell \& Lynden-Bell 1995). The distribution of distant halo globular clusters like Pal I has been used to reinforce the existence of both groups (Kunkel 1979; Majewski 1994).

Lynden-Bell (1976) speculated that if the LMC and SMC are physically associated then it is possible that they were once part of a larger system. He noted that Draco and Ursa Minor appeared to be distributed on the sky along a great circle but opposite the Magellanic Clouds, suggesting that they were debris torn off the so-called Greater Magellanic Galaxy. A later study showed that the elongation of Draco and Ursa Minor occurs along the Magellanic Stream (Lynden-Bell 1982). If the Clouds were at more than one pericentric passage around the MW, on the previous passage Sculptor and perhaps Draco and Ursa Minor were pulled out, but the next pericentric passage has been more severe with the two Clouds being pulled apart.

More recently it has been proposed that the Magellanic Clouds were accreted into the MW halo as the largest members of a group of dwarf galaxies, named the Magellanic Group, that fell into the MW halo at a relatively recent time (D'Onghia \& Lake 2008). This scenario is motivated by theoretical and observational arguments. It is naturally expected in the most popular scenario of structure formation, the $\Lambda$ cold dark matter (CDM) model, which predicts that the abundance of sub-structures is self-similar. This means that an abundance of sub-halos is expected at all observable mass scales. Thus, if hundreds of satellite halos are predicted to surround the MW halo (Klypin et al. 1999, Moore et al. 1999), at lower mass scales dwarf-galaxy halos can also host sub-structures. The second motivation comes from the discovery of nearby associations of dwarf galaxies at distances of $1-3 \mathrm{Mpc}$ from the Local Group (Tully et al. 2006). These systems are defined as associations, rather than groups, because of their low density and and the fact that they are loosely bound systems - not in a dynamical equilibrium - since the crossing time is very long: $80 \%$ of the Hubble time. They have masses ranging between $5 \cdot 10^{10} \mathrm{M}_{\odot}$ and $1 \cdot 10^{11} \mathrm{M}_{\odot}$ and typically contain a larger dwarf irregular galaxy and a few dwarfs comparable in mass to dwarf spheroidals, as well as fainter satellite galaxies. NGC 3109 is one example of such association, with a dwarf irregular as the largest member surrounded by a few fainter dwarf galaxies, including Antlia and the recently discovered fainter system Antlia B (Sand et al. 2015).

The LMC may have been the largest member of such a dwarf association. In this scenario, when this group fell into the MW potential, it carried several of the bright satellites of the MW, such as Draco, Sculptor, Sextans, Ursa Minor, as well as Sagittarius, Sextans and Leo II, among others. This picture is supported by the evidence that in the hierarchical universe many satellite halos potentially hosting dwarf galaxies have been accreted into the MW as pairs (Sales et al. 2007, Ludlow et al. 2009; Klimentowski et al. 2010) or as part of multiple systems, as indicated by cosmological simulations (Lux et al. 2010, D'Onghia \& Lake 2008; Li \& Helmi 2008; Ludlow et al. 2009; Wetzel et al. 2015).

Observationally, the discovery of conformity positions and velocities of satellite pairs, such as LMC, SMC, Leo IV and Leo V (Belokurov et al. 2008), and of satellites close to the Sagittarius stream, e.g. Segue 1 (Belokurov et al. 2007, Niederste-Ostholt et al. 2009), Bootes II (Koch et al. 2009), Segue 2 (Belokurov et al. 2009) and Segue 3 (Belokurov et al. 2010) further motivated the recent search for associations between satellite galaxies and 
streams.

Recent studies examined the orbits of the bright dwarf galaxies bound to the LMC in the past as part of a Magellanic Group falling into the MW at its first or second perigalacticon, but reported contrasting results. By running a Monte Carlo suite of models of the Magellanic Group in gravitational interaction with the MW and comparing the results to the available kinematic data for the local dwarf galaxies, Nichols et al. (2011) concluded that Draco, Sculptor, Sextans, Ursa Minor, and Sagittarius are consistent with having fallen in along with the Magellanic System, in addition to cases such as Carina and Leo I, which might have a different origin. A different study examined the infall of a Magellanic Group into the MW using zoom-in high-resolution $N$-body simulations of a MW-sized halo (Sales et al. 2011). This study finds that Draco and Ursa Minor are unlikely to be accreted into the MW as part of the Magellanic Group if the Clouds are on their first perigalacticon, although there could possibly remain a large number of faint satellites near the Clouds.

The recent discovery of several new ultra-faint MW companions, using the data from the Dark Energy Survey (DES), is consistent with the scenario of a Magellanic Group (see Figure 8. Koposov et al. 2015, Bechtol et al. 2015, Martin et al. 2015, Westmeier et al. 2015). Three systems qualify to be dwarf-galaxy candidates, based on their morphological properties, while the nature of the other six objects is currently uncertain. In particular, Reticulum 2, Horologium 1, and Eridanus 3 are aligned with the LMC's orbital plane and may form part of the its cortege. Similarly, Tucana 2, Phoenix 2 and Grus 1 appear to align with the SMC. This suggests a picture in which at least some newly discovered ultra-faint dwarf galaxies, together with some of the already well-known satellites, belonged to a loose association of dwarfs bound to the LMC and SMC, with size and mass comparable to the dwarf associations discovered around the Local Group.

There is some evidence that satellite galaxies are not isotropically distributed around massive galaxies (Bailin et al. 2008 and reference therein), and correlations among their spatial distribution or possible alignments are ubiquitous. Indeed, half of the satellite galaxies of M31 may lie in a thin, extended and rotating plane (Ibata et al. 2013), although these findings are still uncertain (Phillips et al. 2015). Planes of satellites might be present in the Centaurus Group as well (Tully et al. 2015).

If there was a Magellanic Group of dwarf galaxies, the latest measurements of the proper motion of the LMC (K3) would suggest that it is currently either at its first perigalacticon or has already completed a full orbit.

Kinematic data is inconclusive as to whether the LMC is on its first passage or has already completed a full orbit (Sect. 3.1). If at its first perigalacticon, then most of its associated satellite dwarf galaxies should be tightly clustered around its location. Although rare, some LMC-associated systems may still be found well away from the LMC but along the orbital path of the Magellanic Group (Sales et al. 2011, Deason et al. 2015). Of the well-known MW satellites, only the SMC is unambiguously associated with the LMC. There is some chance that Fornax, Carina and Sculptor might be associated, although the available proper motions would indicate that the orbital planes of these three satellites might not be aligned with that of the Clouds. If the LMC has already completed one full orbit, then several additional dwarfs qualify for association. Leo II, Leo IV and Leo V, in particular, show strong spatial and velocity coincidence with the tidal debris from LMC, making them candidates for past association with the LMC, in addition to Canes Venatici II. These tentative associations of the known dwarf galaxies of the MW to a Magellanic Group will be confirmed or ruled out when their proper motions become available. 


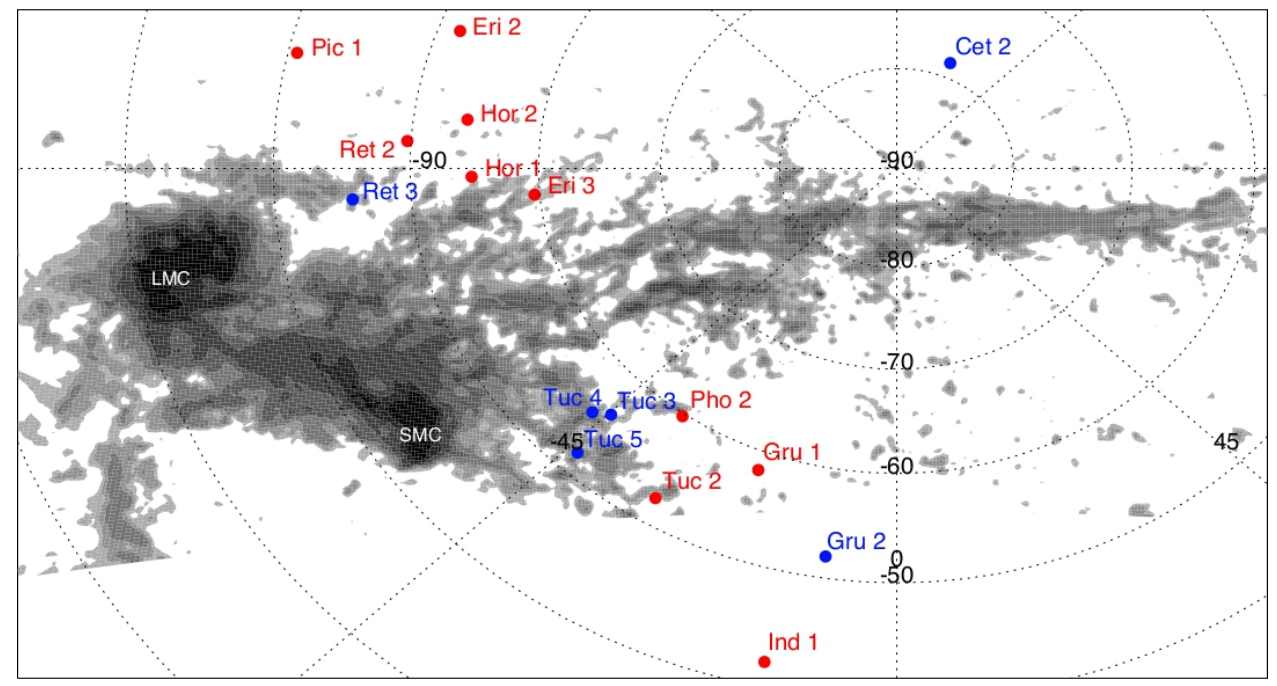

Figure 8

Location of the MW satellite galaxies with respect to the H I Stream, including recently detected ultra-faint dwarfs found in the DES Survey Year 1 data (red) and Year 2 data (blue). Credit: Vasily Belokurov, updated from Koposov et al. (2015) with H I data from Putman et al. (2003b).

If a Magellanic Group fell into the MW carrying a number of satellites, the SMC might have played a role as well. Recent estimates of the star-formation history of the SMC, derived using high-resolution color-magnitude diagrams and images from the HST Advanced Camera for Surveys, indicate that the SMC experienced a global peak of star formation activity between 5-7 Gyr ago (Cignoni et al. 2013). It should be noted that this result is consistent with the sudden appearance 7-8 Gyr ago of an enhancement of globular clusters in the SMC (Piatti 2011).

If a dramatic event happened in the $\mathrm{SMC} \sim 7 \mathrm{Gyr}$ ago as suggested by these findings, the question is whether this can constrain the past orbital history of the SMC. Tsujimoto \& Bekki (2009) proposed that the SMC experienced a merger event 7.5 Gyr ago in a small group of gas-rich dwarfs with the size and mass comparable to the Clouds. While it is unclear whether this picture can reproduce the SMC age-metallicity relation reported by Cignoni et al. (2013), it seems an appealing scenario to be explored with future numerical simulations, especially in light of the possibility that the Magellanic Clouds may have been accreted as a group of dwarfs instead of a galaxy pair.

The discussion on the past existence of the Magellanic Group should end with a note of caution. Despite the fact that satellite galaxies appear to be distributed on the sky along a great circle rather than in a spherical way, the great circle has a polar orientation relative to the MW disk. The discovery of most of the SDSS ultra-faint dwarfs in the northern hemisphere might have increased the apparent significance of the alignment. Another important point about associations or planes of dwarfs is that they affect the statistics. If satellites are accreted as groups rather than individuals possible alignments are more likely to be spotted. Thus, the completion of the DES survey, by covering areas farther away from the polar structure, as well as Pan-STARRS and the next generation of deep wide-field surveys, will be able to characterize and fully address the question of the anisotropy of the 
distribution of MW satellites. Furthermore, all of these newly-discovered objects trail the LMC and SMC in their orbits in the sky. In the Magellanic Group scenario, there should also be a population of satellites that leads the LMC and SMC; such a population awaits discovery.

\subsection{Fate of the Magellanic Clouds}

A question closely related to the fate of the Stream is the fate of the Clouds themselves, which depends on whether they are just at their first passage or have already completed an orbit around the MW. An old argument from Tremaine (1976), made with the belief that the Clouds had made multiple passages around the MW, posited that dynamical friction must have caused substantial decay of the orbits of the Magellanic Clouds over the last 10 Gyr, during which time the Galactic tidal force at perigalacticon must have steadily increased. The LMC in this scenario will be disrupted by the Galaxy in 2-4 Gyr, increasing the luminosity of the Galaxy by $-0.24 \mathrm{mag}$. However, these calculations did not account for the tidal field of M31 and the new orbital solutions for the Clouds. Given that the MW is approaching M31 and is expected to merge within it in 4 Gyr (Cox \& Loeb 2008; van den Marel et al. 2012), this calls into question which merger will happen first.

\section{SUMMARY AND FUTURE DIRECTIONS}

\section{SUMMARY POINTS}

1. Via radio, UV, and optical observations, we now have a comprehensive knowledge of the morphological, chemical, structural, and physical properties of the Magellanic Stream, Bridge, and Leading Arm.

2. The recent third-epoch HST high-precision proper motions measurements of the Magellanic Clouds show that the Clouds are either on their first passage onto the MW or on an eccentric long orbit. This differs remarkably from the past canonical picture in which the Clouds are our long-term companions traveling on a quasiperiodic short orbit around the MW.

3. As a consequence of the new orbital history of the Clouds, a new picture has emerged for the origin of the Stream. Observations and theoretical arguments strongly suggest a recent collision between the Clouds in the last 200-300 Myr that formed the Bridge and contributed to the Leading Arm and the trailing Stream. However, the Stream's total mass remains a challenge, particularly given the large component of ionized gas present. Its total mass is underestimated by a factor of $\sim 10-100$ in current models. In addition the detailed observations of the filamentary structure of the Stream indicate that the interaction with the MW gas corona and the ram pressure stripping still play a significant role. Furthermore, there is clear kinematic evidence that the LMC filament of the Stream connects back to the SEHO region of the LMC, which contains the starburst region 30 Doradus. This suggests that feedback from star formation contributes gas to one portion of the Stream.

4. Several basic issues such as whether the LMC and SMC are on their first approach, or bound to each other, or truly associated with other MW satellites, remain at present unresolved, largely due to uncertainties in the total mass of the MW and 
LMC.

5. The Stream offers a valuable case study of the gas accretion processes operating in an $\approx L_{*}$ galaxy halo, where a merger provides gas to fuel future star formation. It is providing copious amounts of gas to the MW's corona. The fate of this gas depends on the disruptive encounter with the coronal gas, and the balance between evaporation and condensation.

\section{FUTURE RESEARCH DIRECTIONS}

1. Ongoing surveys for a stellar component to Stream will address the long-standing question: if it is a tidal feature, where are its stars? The SMASH survey using the DECam camera will map 480 square degrees of the outskirts of the Magellanic Clouds (Nidever 2015), supplementing the 5000 square degrees covered by the Dark Energy Surveys.

2. Sensitive future radio surveys could address questions on the extent of the H I Stream, such as whether its tip crosses the Galactic plane. This will constrain further whether the Cloud are at their first or second past perigalacticon around the MW.

3. More measurements of the metallicity of the Leading Arm and how it compares to the current-day LMC and SMC metallicities will constrain the formation of the Leading Arm. This necessitates finding AGN bright enough for UV observations in Leading Arm directions with substantial H I column densities.

4. Further constraints on the spatial extent of the diffuse outer layers of the Stream will refine our knowledge of its total gas mass. This could be achieved by UV absorption studies of AGN lying far away on the sky from the $21 \mathrm{~cm}$-emitting body of the Stream.

5. Further studies of the relationship between the $\mathrm{H} \alpha$-emitting gas and the UVabsorbing gas will help elucidate the properties of the ionized gas, including the ionization mechanism.

6. Dynamical models based on cosmological simulations with gas dynamics included, will greatly tighten our constraints not only on the dynamics of the LMC-SMC but also on the Local Group. It will be useful to further constrain how the Clouds are accreted into the MW, their past orbital history and hence scenarios for the formation of the Magellanic Stream.

7. The next big improvement in proper-motion measurements for the LMC and SMC will be possible with the Gaia mission.

8. Further $H S T$ proper motion measurements will be extremely useful to better constrain the SMC's internal kinematics, which are currently poorly understood. This will enhance our ability to further constrain theoretical models of the SMC's dynamics.

9. In a few years, the proper motions of distant satellite galaxies in our MW will be measured by the James Webb Space Telescope. This will likely confirm or confute the existence of a Magellanic association of dwarf galaxies.

10. Meanwhile, searches for Stream-analogs in high-redshift galaxies will help reveal 
whether the Magellanic Stream is unusual (in terms of gas mass and spatial extent). We know from SDSS statistics that the MW is somewhat unusual in having two relatively massive dwarf companions so close by (Tollerud et al. 2011). The nearby spirals M31 and M33 are connected by a bridge of H I (Braun \& Thilker 2004, Wolfe et al. 2013), although it is unclear whether this represents a condensing intergalactic filament rather than a tidal feature. More examples of extragalactic gaseous tidal streams are needed to understand the role such features play in the larger picture of galaxy evolution.

We end this article by returning to the Stream's namesake, Ferdinand Magellan. Although Magellan's crew completed the first circumnavigation of the globe when they sailed his ship the Victoria back to Europe in 1522, the voyage ended tragically for him in the Philippines. There is a striking parallel between the around-the-world nature of his voyage and the around-the-galaxy nature of the Magellanic Stream, revealed to us almost 500 years later. Magellan's voyage opened up the world to trade and discovery. His Stream is blazing its own trail around the Galaxy.

\section{DISCLOSURE STATEMENT}

The authors are not aware of any affiliations, memberships, funding, or financial holdings that might be perceived as affecting the objectivity of this review.

\section{ACKNOWLEDGMENTS}

E.D.O. acknowledges support from the Alfred P. Sloan foundation. We thank Jay Gallagher, Julio Navarro, Mary Putman, Snezana Stanimirović, Carlos Vera-Ciro, Bart Wakker, and Dennis Zaritsky for useful comments, and Joss Bland-Hawthorn for an insightful review. We thank Kat Barger, Gurtina Besla, Francois Hammer, Nitya Kallivayalil, and David Nidever for permission to use their graphics, Stephen Pardy for providing Figure 7, and Vasily Belokurov for providing Figure 8.

\section{LITERATURE CITED}

1. Bagheri G, Cioni MRL, Napiwotzki R 2013, Astron. Astrophys., 551:A78

2. Bailin J, Power C, Norberg P, Zaritsky D, Gibson BK 2008, MNRAS, 390:1133

3. Bajaja E, Cappa de Nicolau CE, Cersosimo JC, et al. 1985, Ap. J. Suppl., 58:143

4. Barger KA, Haffner LM, Bland-Hawthorn J 2013, Ap. J., 771:132

5. Barnes DG, Staveley-Smith L, de Blok WJG, et al. 2001, MNRAS, 322:486

6. Bechtol K, Drlica-Wagner A, Balbinot E, et al. 2015, Ap. J., 807:50

7. Bekki K, Chiba M 2005, MNRAS, 356:680

8. Bekki K, Chiba M 2007, Publ. Astron. Soc. Aust., 24:21

9. Bekki K, Stanimirović S 2009, MNRAS, 395:342

10. Bekki K, Couch WJ, Beasley MA, et al. 2004, Ap. J. Lett., 610:L93

11. Belokurov V, Koposov S 2015, MNRAS, submitted (arXiv:1511.03667)

12. Belokurov V, Zucker DB, Evans NW, et al. 2007, Ap. J., 654:897

13. Belokurov V, Walker MG, Evans NW, et al. 2008, Ap. J. Lett., 686:L83

14. Belokurov V, Walker MG, Evans NW, et al. 2009, MNRAS, 397:1748 
15. Belokurov V, Walker MG, Evans NW, et al. 2010, Ap. J. Lett., 712:L103

16. Besla G, Kallivayalil N, Hernquist L, et al. 2007, Ap. J., 668:949

17. Besla G, Kallivayalil N, Hernquist L, et al. 2010, Ap. J. Lett., 721:L97

18. Besla G, Hernquist L, Kallivayalil N, et al. 2012, MNRAS, 421:2109

19. Bland-Hawthorn J, Maloney PR 1999, Ap. J. Lett., 510:L33

20. Bland-Hawthorn J, Maloney PR 2002, ASPC, 254:267

21. Bland-Hawthorn J, Sutherland RS, Agertz O, Moore B 2007, Ap. J. Lett., 670:L109

22. Bland-Hawthorn J, Maloney P, Sutherland RS, Madsen GJ 2013, Ap. J., 778:58

23. Boylan-Kolchin M, Besla G, Hernquist L 2011, MNRAS, 414:1560

24. Braun R, Thilker DA 2004, Astron. Astrophys., 417:421

25. Bregman JN, Harrington JP 1986, Ap. J., 309:833

26. Brück MT, Hawkins MRS 1983, Astron. Astrophys., 124:216

27. Brüns C, Kerp J, Kalberla PMW, Mebold U 2000, Astron. Astrophys., 357:120

28. Brüns C, Kerp J, Staveley-Smith L, et al. 2005, Astron. Astrophys., 432:45

29. Busha MT, Alvarez MA, Wechsler RH, Abel T, Strigari LE 2010, Ap. J., 710:408

30. Caldwell JAR, Coulson IM 1986, MNRAS, 218:223

31. Casetti-Dinescu DI, Moni Bidin C, Girard RM, et al. 2014, Ap. J. Lett., 784:L37

32. Chomiuk L, Povich MS 2011, Ap. J., 142:197

33. Cignoni M, Cole AA, Tosi M, et al. 2013, Ap. J., 775:83

34. Cioni MRL, Habing HJ, Israel FP 2000, Astron. Astrophys. Lett., 358:L9

35. Cioni MRL 2009, Astron. Astrophys., 506:1137

36. Cohen RJ 1982, MNRAS, 199:281

37. Cole AA, Tolstoy E, Gallagher JS, Smecker-Hane A 2005, Astron. J. 129:1465

38. Collins JA, Shull JM, Giroux ML 2005, Ap. J., 623:196

39. Connors TW, Kawata D, Maddison, ST, Gibson BK 2004, Publ. Astron. Soc. Aust., 21:222

40. Connors TW, Kawata D, Bibson BK 2006, MNRAS, 371:108

41. Costa E, Méndez RA, Pedreros MH, et al. 2011, Astron. J, 141:136

42. Cox TJ, Loeb A 2008, MNRAS, 386:461

43. Davies RD, Wright AE 1977, MNRAS, 180:71

44. de Vaucouleurs G, Freeman KC 1972, Vistas in Astronomy, 14:163

45. Deason AJ, Wetzel AR, Garrison-Kimmel S, Belokurov V 2015, MNRAS, 453:3568

46. Demers S, Irwin MJ 1991, Astron. Astrophys. Suppl., 91:171

47. Diaz JD, Bekki K 2011a, Publ.. Astron. Soc. Aust., 28:117

48. Diaz JD, Bekki K 2011b, MNRAS, 413:2015

49. Diaz JD, Bekki K 2012, Ap. J., 750:36

50. Dieter NH 1965, Astron. J., 70:552

51. Dieter NH 1971, Astron. Astrophys., 12:59

52. Dobbie PD, Cole AA, Subramaniam A, Keller S 2014, MNRAS, 442:1680

53. D'Onghia E, Lake G 2008, Ap. J. Lett., 686:L61

54. D'Onghia E, Besla G, Cox TJ, Hernquist L 2009, Nature, 460:605

55. D’Onghia E, Vogelsberger M, Faucher-Giguere, C-A, Hernquist L 2010, Ap. J., 725:353

56. Dopita MA, Lawrence CJ, Ford HC, Webster BL 1985, Ap. J., 296:390

57. Drake AJ, Cook KH, Alcock C, 2001, Bull. Am. Astron. Soc., 33:1379

58. Fong R, Jones LR, Shanks T, Stevenson PRF, Strong AW 1987, MNRAS, 224:1059

59. For BQ, Staveley-Smith L, Matthews D, McClure-Griffiths NM 2014, Ap. J., 792:43

60. Fox AJ, Wakker BP, Savage BD, et al. 2005, Ap. J., 630:332

61. Fox AJ, Wakker BP, Smoker JV, et al. 2010, Ap. J., 718:1046

62. Fox AJ, Richter P, Wakker BP, et al. 2013, Ap. J., 772:110

63. Fox AJ, Wakker BP, Barger KA, et al. 2014, Ap. J., 787:147

64. Fox AJ, Bordoloi R, Savage, BD et al. 2015 Ap. J. Lett., 799:L7

65. Fraternali F, Marasco A, Marinacci F, Binney J 2013, Ap. J. Lett., 764:L21 
66. Fraternali F, Marasco A, Armillotta L, Marinacci F 2015, MNRAS Lett., 447:L70

67. Fujimoto M, Sofue Y 1976, Astron. Astrophys., 47:263

68. Ganguly R, Sembach KR, Tripp TM, Savage BD 2005, Ap. J. Suppl., 157:251

69. Gardiner LT, Hatzidimitriou D 1992, MNRAS, 257:195

70. Gardiner LT, Sawa T, Fujimoto M 1994, MNRAS, 266:567

71. Gardiner LT, Noguchi M 1996, MNRAS, 278:191

72. Gibson BK, Giroux ML, Penton SV, et al. 2000, Astron. J., 120:1803

73. Gnat O, Sternberg A 2009, Ap. J., 693:1514

74. Gnat O, Sternberg A, McKee CF 2010, Ap. J., 718:1315

75. Gomez FA, Besla G, Carpintero DD, et al. 2015, Ap. J., 802:128

76. Graczyk D, Pietrzyński G, Thompson IB, et al. 2014, Ap. J., 780:59

77. Grocholski AJ, Cole AA, Sarajedini A, Geisler D, Smith VV 2006, Ap. J., 132:1630

78. Guglielmo M, Lewis GF, Bland-Hawthorn J 2014, MNRAS, 444:1759

79. Guhathakurta P, Reitzel DB 1998, ASP Conf. Ser, 136:22

80. Hammer F, Yang YB, Flores H, Puech M, Fouquet S 2015, Ap. J., in press (arXiv:1510.00096)

81. Harris J, Zaritsky D 2004, Astron. J., 127:1532

82. Harris J, Zaritsky D 2006, Astron. J., 131:2514

83. Harris J, Zaritsky D 2009, Astron. J., 138:1243

84. Harris J 2007, Ap. J., 658:345

85. Haynes MP 1979, Astron. J., 84:1173

86. Heitsch F, Putman ME 2009, Ap. J., 698:1485

87. Heller P, Rohlfs K 1994, Astron. Astrophys., 291:743

88. Hilditch RW, Howarth ID, Harries TJ 2005, MNRAS, 357:304

89. Hindman JV, Kerr FJ, McGee RX 1963, Aust. J. Physics, 16:570

90. Hsu WH, Putman ME, Heitsch F, et al. 2011, Astron. J., 141:57

91. Hulsbosch ANM 1968, Bull. Astr. Inst. Netherlands, 20:33

92. Hulsbosch ANM, Raimond E 1966, Bull. Astr. Inst. Netherlands, 18:413

93. Hulsbosch ANM, Wakker BP 1988, Astron. Astrophys. Suppl., 75:191

94. Ibata RA, Lewis GF, Conn AR, et al. 2013, Nature, 493:62

95. Irwin MJ, Demers S, Kunkel WE 1990, Astron. J., 99:191

96. Jones BF, Klemola AR, Lin DNC 1994, Astron. J., 107:1333

97. Joung MR, Bryan GL, Putman ME 2012, Ap. J., 745:148

98. Kalberla PMW, Haud U 2006, Astron. Astrophys., 455:481

99. Kalberla PMW, Burton WB, Hartmann D, et al. 2005 Astron. Astrophys., 440:775

100. Kallivayalil N, van der Marel RP, Alcock C, et al. 2006a, Ap. J., 638:772

101. Kallivayalil N, van der Marel RP, Alcock C, et al. 2006b, Ap. J., 652:1213

102. Kallivayalil N, van der Marel RP, Besla G, Anderson J, Alcock C 2013, Ap. J., 764:161

103. Kerr FJ, Hindman JV, Robinson BJ 1954, Aust. J. Phys., 7:297

104. Kennicutt RC, Bresolin F, Bomans DJ, Bothun GD, Thompson IB 1995, Astron. J., 109:594

105. Kim S, Staveley-Smith L, Dopita MA, et al. 1998, Ap. J., 503:674

106. Klimentowski J, Łokas EL, Knebe A, et al. 2010, MNRAS, 402:1899

107. Klypin A, Kravtsov AV, Valenzuela O, Prada F 1999, Ap. J., 522:82

108. Kobulnicky HA, Dickey JM 1999, Astron. J., 117:908

109. Koch A, Wilkinson MI, Klenya JT, et al. 2009, Ap. J., 690:453

110. Koposov SE, Belokurov V, Torrealba G, Evans NW 2015, Ap. J., 805:130

111. Kroupa P, Röser S, Bastian U 1994, MNRAS, 266:412

112. Kroupa P, Bastian U 1997, New Astronomy, 2:77

113. Kumari N, Fox AJ, Tumlinson J, et al. 2015, Ap. J., 800:44

114. Kunkel WE 1979, Ap. J., 228:718

115. Kunkel WE, Demers S, Irwin MJ, Albert L 1997, Ap. J. Lett., 488:L129

116. Kunkel WE, Demers S, Irwin MJ 2000, Astron. J., 119:2789 
117. Kwak K, Shelton RL 2010, Ap. J., 719:523

118. Kwak K, Henley DB, Shelton RL 2011, Ap. J., 739:30

119. Lehner N 2002, Ap. J., 578:126

120. Lehner N, Howk JC, Keenan FP, Smoker JV 2008, Ap. J., 678:219

121. Lehner N, Howk JC 2011, Science, 334:955

122. Li Y-S, Helmi A 2008, MNRAS, 385:1365

123. Li Y-S, White SDM 2008, MNRAS, 384, 1459

124. Lin DNC, Lynden-Bell D 1977, MNRAS, 181:59

125. Lin DNC, Lynden-Bell D 1982, MNRAS, 198:707

126. Lin DNC, Jones BF, Klemola AR 1995, Ap. J., 439:652

127. Lockman FJ, Murphy EM, Petty-Powell S, Urick VJ 2002, Ap. J., 140:331

128. Łokas EL, Semczuk M, Gajda G, D’Onghia E. 2015, Ap. J., 810:100

129. Lu L, Savage BD, Sembach KR 1994, Ap. J. Lett., 437:L119

130. Lu L, Sargent WLW, Savage BD, et al. 1998, Astron. J., 115:162

131. Ludlow AD, Navarro JF, Springel V, et al. 2009, Ap. J., 692:931

132. Lux H, Read JI, Lake G 2010, MNRAS, 406:2312

133. Lynden-Bell D 1976, MNRAS, 174:695

134. Lynden-Bell D 1982, Observatory, 102:7

135. Lynden-Bell D, Lin DNC 1977, MNRAS, 181:37

136. Lynden-Bell D, Lynden-Bell RM 1995, MNRAS, 275:429

137. Mackey D, Koposov SE, Erkal D, et al. 2015, MNRAS, submitted (arXiv:1508.01356)

138. Majewski SR 1994, Ap. J. Lett., 431:L17

139. Maragoudaki F, Kontzias M, Morgan DH, et al. 2001, Astron. Astrophys., 379:864

140. Marasco A, Marinacci F, Fraternali F 2013, MNRAS, 433:1634

141. Marinacci F, Binney J, Fraternali F, et al. 2010, MNRAS, 404:1464

142. Martin NF, Nidever DL, Besla G, et al. 2015, Ap. J. Lett., 804:L5

143. Massey P, Olsen KAG 2003, Astron. J., 126:2867

144. Mastropietro C, Moore B, Mayer L, Wadsley J, Stadel J 2005, MNRAS, 363:509

145. Mathewson DS 1985, Publ. Astron. Soc. Aust., 6:104

146. Mathewson DS, Ford VL 1984, in Structure and Evolution of the Magellanic Clouds, eds. van den Bergh S, de Boer KS, 125-136 (Kluwer, Dordrecht)

147. Mathewson DS, Cleary MN, Murray JD 1974, Ap. J., 190:291

148. Mathewson DS, Schwarz MP, Murray JD 1977, Ap. J. Lett., 217:L5

149. Matthews D, Staveley-Smith L, Dyson P, Muller E 2009, Ap. J. Lett., 691:L115

150. Mayer L, Governato F, Colpi M, et al. 2001, Ap. J., 559:754

151. McClure-Griffiths NM, Staveley-Smith L, Lockman FJ, et al. 2008, Ap. J. Lett., 673:L143

152. McClure-Griffiths NM, Pisano DJ, Calabretta MR, et al. 2009 Ap. J. Suppl., 181:398

153. McClure-Griffiths NM, Madsen GJ, Gaensler BM, McConnell D, Schnitzeler DHFM 2010, Ap. $J ., 725: 275$

154. McGee RX, Newton LM 1986, Proc. Astron. Soc. Austral., 6:471

155. McMillan PJ 2011, MNRAS, 414:2446

156. Mellinger A 2009, PASP, 121:1180

157. Meurer GR, Bicknell GV, Gingold RA 1985, Publ. Astron. Soc. Aust., 6:195

158. Mirabel IF 1981, Ap. J., 250:528

159. Mirabel IF, Cohen RJ, Davies RD 1979, MNRAS, 186:433

160. Misawa T, Charlton JC, Kobulnicky HA, Wakker BP, Bland-Hawthorn J 2009, Ap. J., 695:1382

161. Mizuno N, Muller E, Maeda H, et al. 2006, Ap. J. Lett., 643:L107

162. Moore B, Davis M 1994, MNRAS, 270:209

163. Moore B, Ghigna S, Governato F, et al. 1999, Ap. J. Lett., 524:L19

164. Morras R 1983, Astron. J., 88:62 
165. Morras R, Bajaja E, Arnal EM, Pöppel WGL 2000, Astron. Astrophys. Suppl., 142:25

166. Muller E, Staveley-Smith L, Zealey WJ 2003, MNRAS, 338:609

167. Murai T, Fujimoto M 1980, Proc. Astron. Soc. Japan, 32:581

168. Murali C 2000, Ap. J. Lett., 529:L81

169. Murray CE, StanimiroviŞ, McClure-Griffiths NM, et al. 2015, Ap. J., 808:41

170. Nichols M, Colless J, Colless M, Bland-Hawthorn J 2011, Ap. J, 742:110

171. Niederste-Ostholt M, Belokurov V, Evans NW, et al. 2009, MNRAS, 398:1771

172. Navarro JF, Frenk CS, White SDM 1997, Ap. J., 490:493

173. Nidever DL 2015, ASP Conference Series, 491:325

174. Nidever DL, Majewski SR, Burton WB 2008, Ap. J., 679:432

175. Nidever DL, Majewski SR, Burton WB, Nigra L 2010, Ap. J., 723:1618

176. Nigra L, Stanimirović S, Gallagher JS, et al. 2012, Ap. J., 723:48

177. Noël, NED, Conn BC, Carrera R, et al. 2013, Ap. J., 768:109

178. Olsen KAG, Massey P 2007, Ap. J. Lett., 656:L61

179. Olsen KAG, Zaritsky D, Blum RD, Boyer ML, Gordon KD 2011, Ap. J., 737:29

180. Pagel BEJ, Edmunds MG, Fosbury RAE, Webster BL 1978, MNRAS, 184:569

181. Pagel BEJ, Tautvaisiene G 1998, MNRAS, 299:535

182. Pedreros MH, Anguita C, Maza J 2002, Astron. J., 123:1971

183. Peek JEG, Putman ME, McKee CF, Heiles C, Stanimirović S 2007, Ap. J., 656:907

184. Phillips JI, Cooper MC, Bullock JS, Boylan-Kolchin M 2015, MNRAS, 453:3839

185. Piatek S, Pryor C, Olszewski EW 2008, Astron. J., 135:1024

186. Piatti AE 2011, MNRAS Lett., 418:L69

187. Pietrzyński G, Graczyk D, Gieren W, et al. 2013, Nature, 495:76

188. Peebles PJE, Tully RB 2013, Ap. J., 778:137

189. Prevot L, Andersen J, Ardeberg A, et al. 1985, Astron. Astrophys. Suppl., 62:23

190. Putman ME 2000, Publ. Astron. Soc. Aust., 17:1

191. Putman ME, Gibson BK, Staveley-Smith L, et al. 1998, Nature, 394:752

192. Putman ME, Bland-Hawthorn J, Veilleux S, et al. 2003a, Ap. J., 597:948

193. Putman ME, Staveley-Smith L, Freeman KC, Gibson BK, Barnes DG 2003b, Ap. J., 586:170

194. Putman ME, Peek JEG,, Young MR 2012, Ann. Rev. Astron. Astrophys., 50:491

195. Quilis V, Moore B 2001, Ap. J. Lett, 555:L95

196. Recillas-Cruz E 1982, MNRAS, 201:473

197. Richter P, Sembach KR, Wakker BP, Savage BD 2001, Ap. J. Lett., 562:L181

198. Richter P, Charlton JC, Fangano APM, Ben Bekhti N, Masiero JR 2009, Ap. J., 695:1631

199. Richter P, Fox AJ, Wakker BP, et al. 2013, Ap. J., 772:111

200. Robitaille TP, Whitney BA 2010, Ap. J. Lett., 710:L11

201. Rolleston WRJ, Trundle C, Dufton PL 2002, Astron. Astrophys., 396:53

202. Russell SC, Dopita MA 1992, Ap. J., 384:508

203. Růžička A, Theis C, Palouš J 2009, Ap. J., 691:1807

204. Růžička A, Theis C, Palouš J 2010, Ap. J., 725:369

205. Salem M, Besla G, Bryan G, et al. 2015, Ap. J., submitted, (arXiv:1507.07935)

206. Sales LV, Navarro JF, Lambas DG, White SDM, Croton DJ 2007, MNRAS, 382:1901

207. Sales LV, Navarro JF, Cooper AP, et al. $2011 M N R A S, 418: 648$

208. Sand DJ, Crnojević D, Bennet P, et al. 2015, Ap. J., 806:95

209. Savage BD, Wakker BP, Jannuzi BT, et al. 2000, Ap. J. Suppl., 129:563

210. Schönrich R, Binney J, Dehnen W 2010, MNRAS, 403:1829

211. Sembach KR, Howk JC, Savage BD, Shull JM 2001, Astron. J., 121:992

212. Sembach KR, Gibson BK, Fenner Y, Putman ME 2002, Ap. J., 572:178

213. Sembach KR, Wakker BP, Savage BD, et al. 2003, Ap. J. Suppl., 146:165

214. Shattow G, Loeb A 2009, MNRAS Lett., 392:L21

215. Shull JM, Jones JR, Danforth CW, Collins JA 2009, Ap. J., 699:754 
216. Skowron DM, Jacyszyn AM, Udalski A, et al. 2014, Ap. J., 795:108

217. Smith MC, Ruchti GR, Helmi A, et al. 2007, MNRAS, 379:755

218. Smoker JV, Keenan FP, Polatidis AG, et al. 2000, Astron. Astrophys., 363:451

219. Smoker JV, Keenan FP, Thompson HMA, et al. 2005 Astron. Astrophys., 443:525

220. Songaila A 1981, Ap. J. Lett., 243:L19

221. Sparke LS, Gallagher JS 2000, Galaxies in the Universe, Cambridge: Cambridge University Press

222. Stanimirović S, Staveley-Smith L, Dickey JM, Sault RJ, Snowden SL 1999, MNRAS, 302:417

223. Stanimirović S, Dickey JM, Krčo M, Brooks AM 2002, Ap. J., 576:773

224. Stanimirović S, Staveley-Smith L, Jones PA 2004, Ap. J., 604:176

225. Stanimirović S, Hoffman S, Heiles C, et al. 2008, Ap. J., 680:276

226. Staveley-Smith L, Sault RJ, Hatzidimitriou D, Kesteven MJ, McConnell D 1997, MNRAS, 289:225

227. Staveley-Smith L, Kim S, Calabretta MR, Haynes RF, Kesteven MJ 2003, MNRAS, 339:87

228. Su M, Slatyer TR, Finkbeiner DP 2010, Ap. J., 724:1044

229. Subramaniam A, Subramanian S 2009, Ap. J. Lett., 703:L37

230. Tepper-Garcia T, Bland-Hawthorn J, Sutherland RS 2015, Ap. J., 813:94

231. Tollerud EJ, Boylan-Kolchin M, Barton EJ, Bullock JS, Trinh CQ 2011, Ap. J., 738:102

232. Tolstoy E, Hill V, Tosi M 2009, Ann. Rev. Astron. Astrophys., 47:371

233. Toomre A, Toomre J 1972, Ap. J., 178:623

234. Tremaine SD 1976, Ap. J., 203:72

235. Tsujimoto T, Bekki K 2009, Ap. J. Lett., 700:L69

236. Tully RB, Rizzi L, Dolphin AE, et al. 2006, Astron. J., 132:729

237. Tully RB, Libeskind NI, Karachentsev ID, et al. 2015, Ap. J. Lett., 802:L25

238. Udalski A, Szymanski MK, Soszynski I, Poleski R 2008, Acta Astronomica, 58:69

239. van der Marel RP 2001, Astron. J., 122:1827

240. van der Marel RP, Fardal M, Besla G, et al. 2012, Ap. J., 753:8

241. van der Marel RP, Kallivayalil N 2014, Ap. J., 781:121

242. van der Marel RP, Alves DR, Hardy E, Suntzeff NB 2002, Astron. J., 124:2639

243. van Kuilenburg J 1972, Astron. Astrophys., 16:276

244. Venzmer MS, Kerp J, Kalberla PMW 2012, Astron. Astrophys., 547:12

245. Vieira K, Girard TM, van Altena WF, et al. 2010, Astron. J., 140:1934

246. Wakker BP 2001, Ap. J. Suppl., 136:463

247. Wakker BP, Oosterloo TA, Putman ME 2002, Astron. J., 123:1953

248. Wakker BP, Savage BD, Fox AJ, Benjamin RA, Shapiro PR 2012, Ap. J., 749:157

249. Walker AR 2012, Ap\&sSS, 341:43

250. Wannier P, Wrixon GT 1972, Ap. J. Lett., 173:L119

251. Wayte SR 1989, Publ. Astron. Soc. Aust., 8:195

252. Weiner BJ, Williams TB 1996, Astron. J., 111:1156

253. West KA, Pettini M, Penston MV, Blades JV, Morton DC 1985, MNRAS, 215:481

254. Westmeier T, Koribalski BS 2008, MNRAS Lett., 388:L29

255. Westmeier T, Staveley-Smith L, Calabretta M, et al. 2015, MNRAS, 453:338

256. Wetzel AR, Deason AJ, Garrison-Kimmel S 2015, Ap. J., 807:49

257. Wolfe SA, Pisano DJ, Lockman FJ, McGaugh SS, Shaya EJ 2013, Nature, 497:224

258. Yagi M, Komiyama Y, Yoshida M 2012, Ap. J. Lett., 749:L2

259. Yoshizawa AM, Noguchi M 2003, MNRAS, 339:1135

260. Yozin C, Bekki K 2014, MNRAS, 439:1948

261. Zaritsky D, Harris J, Grebel EK, Thompson IB 2000, Ap. J. Lett., 534:L53

262. Zaritsky D 2004, Ap. J. Lett., 614:L37

263. Zhao HS, Evans NW 2000, Ap. J. Lett., 545:L35 